\title{
MULTI-ELEMENT ABUNDANCE MEASUREMENTS FROM MEDIUM-RESOLUTION SPECTRA. IV. ALPHA ELEMENT DISTRIBUTIONS IN MILKY WAY SATELLITE GALAXIES*
}

\author{
Evan N. Kirby ${ }^{1,5}$, Judith. G. Cohen ${ }^{1}$, Graeme H. Smith ${ }^{2}$, Steven R. Majewski ${ }^{3}$, Sangmo Tony Sohn ${ }^{4}$, \\ And Puragra Guhathakurta ${ }^{2}$ \\ ${ }^{1}$ California Institute of Technology, 1200 East California Boulevard, MC 249-17, Pasadena, CA 91125, USA \\ ${ }^{2}$ University of California Observatories/Lick Observatory, University of California, 1156 High Street, Santa Cruz, CA 95064, USA \\ ${ }^{3}$ Department of Astronomy, University of Virginia, P.O. Box 400325, Charlottesville, VA 22904-4325, USA \\ ${ }^{4}$ Space Telescope Science Institute, 3700 San Martin Drive, Baltimore, MD 21218, USA \\ Received 2010 September 15; accepted 2010 November 16; published 2011 January 5
}

\begin{abstract}
We derive the star formation histories of eight dwarf spheroidal (dSph) Milky Way satellite galaxies from their alpha element abundance patterns. Nearly 3000 stars from our previously published catalog comprise our data set. The average $[\alpha / \mathrm{Fe}]$ ratios for all dSphs follow roughly the same path with increasing $[\mathrm{Fe} / \mathrm{H}]$. We do not observe the predicted knees in the $[\alpha / \mathrm{Fe}]$ versus $[\mathrm{Fe} / \mathrm{H}]$ diagram, corresponding to the metallicity at which Type Ia supernovae begin to explode. Instead, we find that Type Ia supernova ejecta contribute to the abundances of all but the most metal-poor $([\mathrm{Fe} / \mathrm{H}]<-2.5)$ stars. We have also developed a chemical evolution model that tracks the star formation rate, Types II and Ia supernova explosions, and supernova feedback. Without metal enhancement in the supernova blowout, massive amounts of gas loss define the history of all dSphs except Fornax, the most luminous in our sample. All six of the best-fit model parameters correlate with dSph luminosity but not with velocity dispersion, half-light radius, or Galactocentric distance.
\end{abstract}

Key words: galaxies: abundances - galaxies: dwarf - galaxies: evolution - Local Group

Online-only material: color figures

\section{INTRODUCTION}

Understanding the origins of galaxies requires understanding the histories of their dark matter growth, gas flows, and star formation. Of these, the dark matter growth is the most straightforward to model (e.g., Diemand et al. 2007; Springel et al. 2008). The gas flow history presents more difficult obstacles, such as collisional dissipation, gas cooling, stellar feedback, and conversion into stars. Despite the challenges, some models - built on top of dark matter simulations - track all of these processes over cosmic time (e.g., Governato et al. 2007). The results of these models have observational consequences for the properties of the present stellar populations of galaxies.

\subsection{Methods for Determining Star Formation Histories}

The star formation histories (SFHs) of galaxies may be deduced from the colors and magnitudes of the population and from the spectra of the stars and gas, if present. Distant, unresolved galaxies display only a single, composite spectral energy distribution, which may be examined through calibrations of spectrophotometric indices (e.g., Graves \& Schiavon 2008) or, in some cases, spectral synthesis (McWilliam \& Bernstein 2008; Colucci et al. 2009). Nearer stellar systems may be resolved both photometrically and spectroscopically. The Hubble Space Telescope (HST) has enabled the characterization of the SFHs of many nearby galaxies (Weisz et al. 2008; Dalcanton et al. 2009; Bernard et al. 2009), including most of the dwarf galaxies in the Local Group (Holtzman et al. 2006; Orban et al. 2008).

\footnotetext{
* Data herein were obtained at the W. M. Keck Observatory, which is operated as a scientific partnership among the California Institute of Technology, the University of California, and NASA. The Observatory was made possible by the generous financial support of the W. M. Keck Foundation.

5 Hubble Fellow.
}

Photometrically derived SFHs are most sensitive to young stars and metal-rich stars because the separation between isochrones increases with decreasing age and increasing metallicity. Elemental abundances obtained from spectroscopy do not give absolute ages, but they can provide finer relative time resolution for old, metal-poor populations. Gilmore \& Wyse (1991) showed that star formation bursts of varying duration and frequency in dwarf galaxies engrave signatures on the ratio of oxygen to iron as a function of metallicity. Because oxygen-rich Type II supernovae ( $\mathrm{SNe}$ ) explode within tens of Myr of a starburst, the oxygen content of stars forming soon after the burst will be high. Within hundreds of Myr, iron-rich Type Ia SNe begin to explode. The injection of iron into the interstellar medium (ISM) depresses the oxygen-to-iron ratio of subsequently forming stars. These processes are generalizable to other elements. The abundances of the next several elements with even atomic number beyond oxygen - the alpha elements ( $\mathrm{Ne}, \mathrm{Mg}, \mathrm{Si}, \mathrm{S}, \mathrm{Ar}$, $\mathrm{Ca}$, and $\mathrm{Ti}$ - - roughly scale with oxygen abundance. The abundances of iron-peak elements ( $\mathrm{V}, \mathrm{Cr}, \mathrm{Mn}, \mathrm{Co}$, and $\mathrm{Ni}$ ) roughly scale with iron abundance. The trend of the alpha-to-iron-peak ratio with iron-peak abundance, a proxy for elapsed time or integrated star formation, reveals the relative SFH with a resolution of about $10 \mathrm{Myr}$, the approximate timescale for a Type II SN.

\subsection{Chemical Evolution Models}

A glance at a diagram of $[\mathrm{Mg} / \mathrm{Fe}]$ versus $[\mathrm{Fe} / \mathrm{H}]$ gives a qualitative sense of a galaxy's SFH. Converting quantitative abundances into a quantitative SFH requires a chemical evolution model. Pagel (1997) described in detail how to create such a model, and Tolstoy et al. (2009) reviewed recent progress on modeling the SFHs of Local Group dwarf galaxies. Matteucci (2008) described the levels of approximation that the models assume. In general, more sophisticated and presumably more accurate models reduce the number of approximations. The most 
basic assumptions are instantaneous recycling and instantaneous mixing. Consideration of stellar lifetimes and SN delay times removes the first approximation. Three-dimensional hydrodynamical simulations remove the second approximation.

A chemical evolution model reflects the history not only of star formation but also of gas flow. A complete explanation of metallicity and alpha element distributions requires both inflows and outflows. The metallicity distribution functions (MDFs) of nearby Galactic G dwarfs cannot be explained with a closed box model (van den Bergh 1962; Schmidt 1963). Pagel (1997) discussed some of the proposed solutions to the G dwarf problem, including variable nucleosynthesis yields, bimodal star formation, and pre-enrichment. One of the most promising solutions is infalling matter (Larson 1972). Gases undoubtedly flow out of the galaxy, either from SN winds (Mathews \& Baker 1971; Larson 1974) or stripping from the influence of external or host galaxies (Tinsley \& Larson 1979; Lin \& Faber 1983). For example, interactions with the Milky Way (MW) could remove gas from the satellite galaxies discussed here. Both inflows and outflows affect the star formation rate (SFR) throughout the history of the galaxy. Therefore, they shape the MDF and the trend of $[\alpha / \mathrm{Fe}]$ with $[\mathrm{Fe} / \mathrm{H}]$.

Chemical evolution models suffer from uncertainties in the initial mass function (IMF) of stars and stellar lifetimes (Romano et al. 2005), nucleosynthesis yields (Romano et al. 2010), and the delay time distribution (DTD) for Type Ia SNe (Matteucci et al. 2009). However, these limitations have not prevented the models from providing good fits to abundance data. Even models with some of the first theoretical SN yields (Woosley et al. 1993) successfully reproduced the observed metallicity distribution and abundance patterns in the Galaxy (Pagel \& Tautvaišienè 1995). Models with newer SN yields also match the solar neighborhood abundance distributions very well (e.g., Romano et al. 2010). Nonetheless, uncertainties in the model assumptions do complicate the interpretation of the model results. For example, changing the Type Ia DTD, particularly the turn-on time, affects the derived timescale for star formation. The best way to circumvent these uncertainties is to apply the same model consistently to several systems and compare them differentially. Although the absolute ages or SFRs may be affected by systematic errors in the model, the relative quantities between different galaxies will be meaningful.

Local Group dwarf galaxies make good subjects for chemical evolution models. First, the Local Group contains many resolved dwarf galaxies (Mateo 1998; Tolstoy et al. 2009) with stars bright enough for medium- or high-resolution spectroscopy. Second, dwarf galaxies span a wide range of properties, including velocity dispersion and luminosity. The populations of the lowest luminosity galaxies enable the study of star formation on small scales (Martin et al. 2008a; Norris et al. 2008). The changes in populations for more luminous or more massive galaxies show how star formation responds to galaxy size (Mateo 1998; Kirby et al. 2010a). Third, dwarf galaxies host some of the most metal-poor stars known (Kirby et al. 2008, 2009; Geha et al. 2009; Cohen \& Huang 2009, 2010; Frebel et al. 2010a, 2010b; Simon et al. 2010; Norris et al. 2010a, 2010b; Starkenburg et al. 2010; Tafelmeyer et al. 2010). These stars retain the chemical imprint of the ISM when the universe was less than 1 Gyr old. Therefore, dwarf galaxies permit the study of star formation not only on small scales but also at early times. Finally, dwarf galaxies may be the primary building blocks for the MW halo (Searle \& Zinn 1978; White \& Rees 1978). The stellar populations of the surviving dwarf galax- ies may reflect the stellar populations of the dissolved building blocks, and they may show how the surviving satellites evolved since the time of rapid accretion onto the MW.

In a series of articles, Lanfranchi \& Matteucci (2003, 2004, 2007, 2010) and Lanfranchi et al. (2006, 2008) presented numerical models that tracked the evolution of several elements in dwarf spheroidals (dSphs). The models plausibly explained the MDFs and the available multi-element abundance measurements in dSphs. However, large samples of published abundance measurements in any individual dSph have been sparse until recently (Shetrone et al. 2009; Kirby et al. 2009, 2010b; Letarte et al. 2010). Other chemical evolution models of dSphs have examined the effects of reionization (Fenner et al. 2006) and star formation stochasticity (Carigi \& Hernandez 2008). Recchi et al. (2001) constructed one of the first hydrodynamical models of dwarf galaxy evolution. In particular, they simulated a galaxy similar to IZw18. Marcolini et al. (2006, 2008) published hydrodynamical simulations of an isolated, Draco-like dSph. Their models relaxed the assumption of instantaneous mixing and allowed inhomogeneous chemical enrichment. Some of the newest hydrodynamical models (Revaz et al. 2009; Sawala et al. 2010) tracked both the kinematics and abundances of the stars as they form. They attempted to explain not only chemical abundance patterns but also dynamical properties of dSphs, such as the seemingly universal dynamical mass measured within their optical radii (Mateo 1998; Strigari et al. 2008) and out to the edge of their light distributions (Gilmore et al. 2007).

\subsection{History of Chemical Analysis of Milky Way Satellites}

The earliest indications of heavy element abundance spreads among red giants of the dSph systems in Draco, Ursa Minor, Sculptor, and Fornax were first obtained by the multichannel scanner observations of Zinn $(1978,1981)$, initial efforts at spectroscopy (Norris \& Bessell 1978; Kinman \& Kraft 1980; Kinman et al. 1981; Stetson 1984; Smith 1984; Lehnert et al. 1992), and both broad and narrow band photometry (Demers et al. 1979; Smith \& Dopita 1983). The globular clusters (GCs) of the Fornax system proved to differ in their metallicities (Zinn $\&$ Persson 1981). The presence of carbon stars (Aaronson \& Mould 1980; Aaronson et al. 1982, 1983; Azzopardi et al. 1985) and so-called anomalous Cepheids (Demarque \& Hirshfeld 1975; Norris \& Zinn 1975; Hirshfeld 1980; Smith \& Stryker 1986) further indicated the potential complexity of the stellar populations in dSphs. Carbon stars are exceedingly rare in GCs, while the period-luminosity relations of the anomalous Cepheids implied that they are more massive than typical cluster Cepheids (Zinn \& Searle 1976). As a consequence, by the mid-1980s, circumstantial evidence was building to suggest that $\mathrm{dSphs}$ had more complex and possibly more extensive star formation and chemical evolution histories than GCs.

Since that time, the application of ground-based CCD and HST imaging has led to greatly improved color-magnitude diagrams (CMDs) that have clearly shown the presence of significant internal age spreads within some of the MW's retinue of dSphs, such as Carina, Fornax, Leo I, and Sextans (e.g., Mighell 1990, 1997; Smecker-Hane et al. 1996; HurleyKeller et al. 1998; Buonanno et al. 1999; Gallart et al. 1999a, 1999b; Saviane et al. 2000; Lee et al. 2009). Spectroscopy with large ground-based telescopes has demonstrated the presence of abundance inhomogeneities in the majority of these systems (e.g., Suntzeff et al. 1993; Smecker-Hane et al. 1999; Shetrone et al. 2001b, 2003; Tolstoy et al. 2001, 2003, 2004; Winnick 
Table 1

Chemical Evolution Model Variables

\begin{tabular}{|c|c|c|}
\hline Variable & Description & Units \\
\hline$t$ & Time since start of simulation & Gyr \\
\hline$M$ & Mass of a single star & $M_{\odot}$ \\
\hline$\xi_{j}(t)$ & Gas mass in element $j$ & $M_{\odot}$ \\
\hline$X_{j}(t)$ & Mass fraction in element $j$ & Dimensionless \\
\hline$Y$ & Primordial helium mass fraction $\left(X_{\mathrm{He}}(0)\right)$ & Dimensionless \\
\hline$M_{\text {gas }}(t)$ & Total gas mass & $M_{\odot}$ \\
\hline$Z(t)$ & Metal fraction (all elements heavier than $\mathrm{He}$ ) & Dimensionless \\
\hline$\dot{\xi}_{j}(t)$ & Time derivative of $\xi_{j}$ & $M_{\odot} \mathrm{Gyr}^{-1}$ \\
\hline$\dot{\xi}_{j, *}(t)$ & SFR, or rate of gas loss in element $j$ due to star formation & $M_{\odot} \mathrm{Gyr}^{-1}$ \\
\hline$\dot{\xi}_{j, \mathrm{II}}(t)$ & Type II SN or HN yield rate for element $j$ & $M_{\odot} \mathrm{Gyr}^{-1}$ \\
\hline$\epsilon_{\mathrm{HN}}$ & Fraction of $\mathrm{HNe}$ among stars with $M \geqslant 20 M_{\odot}$ & Dimensionless \\
\hline$\zeta_{j, \mathrm{II}}(M, Z)$ & Mass of element $j$ ejected by one Type II SN & $M_{\odot}$ \\
\hline$\dot{\xi}_{j, \mathrm{Ia}}(t)$ & Type Ia SN yield rate for element $j$ & $M_{\odot} \mathrm{Gyr}^{-1}$ \\
\hline$t_{\text {delay }}$ & Type Ia SN delay time & Gyr \\
\hline$\Psi_{\text {Ia }}\left(t_{\text {delay }}\right)$ & Type Ia SN DTD & $\mathrm{SN} \mathrm{Gyr}^{-1} M_{\odot}{ }^{-1}$ \\
\hline$\zeta_{j, \mathrm{Ia}}$ & Mass of element $j$ ejected by one Type Ia SN & $M_{\odot}$ \\
\hline$\dot{\xi}_{j, \mathrm{AGB}}(t)$ & AGB yield rate for element $j$ & $M_{\odot} \mathrm{Gyr}^{-1}$ \\
\hline$\zeta_{j, \mathrm{AGB}}(M, Z)$ & Mass of element $j$ ejected by one AGB star & $M_{\odot}$ \\
\hline$A_{*}$ & Normalization of SFR law (free parameter) & $M_{\odot} \mathrm{Gyr}^{-1}$ \\
\hline$\alpha$ & SFR exponent of $M_{\text {gas }}$ (free parameter) & Dimensionless \\
\hline$A_{\text {in }}$ & Normalization of gas infall rate (free parameter) & $M_{\odot} \mathrm{Gyr}^{-1}$ \\
\hline$\tau_{\text {in }}$ & Gas infall time constant (free parameter) & Gyr \\
\hline$A_{\text {out }}$ & Gas lost per SN (free parameter) & $M_{\odot} \mathrm{SN}^{-1}$ \\
\hline$M_{\text {gas }}(0)$ & Initial gas mass (free parameter) & $M_{\odot}$ \\
\hline
\end{tabular}

2003; Pont et al. 2004; Geisler et al. 2005; McWilliam \& Smecker-Hane 2005a, 2005b; Battaglia et al. 2006; Koch et al. 2006; Bosler et al. 2007; Sbordone et al. 2007; Gullieuszik et al. 2009; Cohen \& Huang 2009, 2010; Kirby et al. 2009).

\subsection{Chemical Evolution Models for the New Catalog}

In this article, we interpret the multi-element abundance distributions in eight dSphs with our own chemical evolution model. The data set is our catalog of abundances based on spectral synthesis of medium-resolution spectra from the DEIMOS spectrograph on the Keck II telescope (Kirby et al. 2010b, hereafter Paper II). The catalog contains 2961 stars with abundance measurements. The number of stars in each $\mathrm{dSph}$ ranges from 141 (Sextans) to 827 (Leo I). It is the largest homogeneous chemical abundance data set in dwarf galaxies. The typical areal coverage is about $300 \mathrm{arcmin}^{2}$ at or near the center of each dSph. The median uncertainty on $[\mathrm{Fe} / \mathrm{H}]$ is 0.12 dex. The fraction of the sample with $[\mathrm{Mg} / \mathrm{Fe}]$ uncertainties less than $0.2(0.3)$ dex is $42 \%(53 \%)$. That fraction increases to $54 \%(69 \%)$ for [Ti/Fe], which is easier to measure than $[\mathrm{Mg} / \mathrm{Fe}]$. For $\langle[\alpha / \mathrm{Fe}]\rangle$ (the average of $[\mathrm{Mg} / \mathrm{Fe}],[\mathrm{Si} / \mathrm{Fe}],[\mathrm{Ca} / \mathrm{Fe}]$, and $[\mathrm{Ti} / \mathrm{Fe}])$, the fraction increases to $71 \%(88 \%)$.

Our one-zone model is simple, but it incorporates some of the newest SN yields and the most recently measured DTD for Type Ia SNe. The biggest advantage of our data set is that it is homogeneous. All of the spectra were obtained with the same spectrograph configuration, and all of the abundances were measured with the same spectral synthesis code. Thus, the derived star formation and gas flow histories from our model-despite its simplicity-will be easy to interpret differentially. In other words, the absolute ages and SFRs may be affected by model uncertainties, but the trends with galaxy properties, such as luminosity, should reflect the true SFHs.

We begin by describing our model (Section 2). Then, we apply the model to the eight dSphs by finding the solution that best matches the abundances. We discuss how our results compare to previous photometric and spectroscopic studies (Section 3). Next, we change some of the model variables to estimate the systematic errors in the derived SFHs (Section 4). Then, we explore how the abundance distributions, SFHs, and gas flow histories change with galaxy properties such as luminosity and velocity dispersion (Section 5). Finally, we enumerate our conclusions (Section 6).

\section{CHEMICAL EVOLUTION MODEL}

In order to provide a rough interpretation of the abundance trends in Paper II's catalog, we have developed a rudimentary model of chemical evolution. Table 1 defines the symbol for each variable or constant in the model. The model supposes that a dwarf galaxy at any instant is a chemically homogeneous system that can accrete or lose gas. The ejecta of Type II SNe enrich the gas according to the total lifetime of massive $(10<$ $\left.M / M_{\odot}<100\right)$ stars, while the Type Ia SNe follow the observed DTD (Maoz et al. 2010; see below). Stars form according to the Kroupa et al. (1993) IMF $\left(d N / d M=0.31 M^{-2.2}\right.$ for $0.5<M / M_{\odot}<1$ and $d N / d M=0.31 M^{-2.7}$ for $\left.M>1 M_{\odot}\right)$.

The calculation tracks the mass of $\mathrm{H}, \mathrm{He}, \mathrm{Mg}, \mathrm{Si}, \mathrm{Ca}, \mathrm{Ti}$, and Fe at each time step $(\Delta t=1 \mathrm{Myr})$. The calculation is terminated when the system reaches zero gas mass.

We define $\xi_{j}(t)$ as the galaxy's gas mass of element $j$ at time $t$. The galaxy's total gas mass at time $t$ is

$$
\begin{gathered}
M_{\mathrm{gas}}(t)=\sum_{j} \xi_{j}(t) \\
\approx \xi_{\mathrm{H}}(t)+\xi_{\mathrm{He}}(t)+20.4\left[\xi_{\mathrm{Mg}}(t)+\xi_{\mathrm{Si}}(t)+\xi_{\mathrm{Ca}}(t)+\xi_{\mathrm{Ti}}(t)\right] \\
+1.07 \xi_{\mathrm{Fe}}(t) .
\end{gathered}
$$

The summation in Equation (1) is over all elements in the periodic table. However, our model tracks only seven elements. 
Therefore, we assume the ratio of the sum of all elements from $\mathrm{Li}$ to $\mathrm{Ti}$, inclusive, to the sum of $\mathrm{Mg}, \mathrm{Si}, \mathrm{Ca}$, and $\mathrm{Ti}$ is the same as in the Sun. This ratio is 20.4 (Anders \& Grevesse 1989). Similarly, we assume the solar ratio for the sum of all elements $\mathrm{V}$ through Ge compared to Fe: 1.07. Elements beyond Ge are neglected. Equation (2) reflects these approximations. For convenience, we define the metallicity of the gas as follows:

$$
Z=\frac{M_{\mathrm{gas}}(t)-\xi_{\mathrm{H}}(t)-\xi_{\mathrm{He}}(t)}{M_{\mathrm{gas}}(t)} .
$$

We also define the gas-phase mass fraction in an element $j$ :

$$
X_{j}(t)=\frac{\xi_{j}(t)}{M_{\mathrm{gas}}(t)} .
$$

The following subsections explain the components of the models. Each component is expressed as the time change in $\xi_{j}(t)$, where $\dot{\xi}_{j} \equiv d \xi_{j}(t) / d t$.

\subsection{Star Formation Rate}

For simplicity, we assume that the SFR is a power law in the gas mass of the galaxy. With this assumption,

$$
\dot{\xi}_{j, *}=A_{*} X_{j}(t)\left(\frac{M_{\mathrm{gas}}(t)}{10^{6} M_{\odot}}\right)^{\alpha} .
$$

The variables $A_{*}$ and $\alpha$ are free parameters in the model. In the complete chemical evolution equation (Equation (16)), the sign of $\dot{\xi}_{j, *}$ is negative because $\xi_{j}$ represents the gas mass, which is depleted due to star formation.

Equation (5) is a generalization of a Kennicutt-Schmidt law (Schmidt 1959; Kennicutt 1998), which connects the SFR to the gas surface density, $\Sigma_{\text {gas }}$. Surface density is perhaps more appropriate for disks than spheroids. Desiring a more threedimensional property, we have used the gas mass, $M_{\text {gas }}$, instead of $\Sigma_{\text {gas }}$. The volume density, $\rho_{\text {gas }}$, would be a better description, but the difference between $M_{\text {gas }}$ and $\rho_{\text {gas }}$ is simply a constant because our model is one-zoned.

\subsection{Type II Supernovae}

In our model, stars more massive than $10 M_{\odot}$ and less massive than $100 M_{\odot}$ explode according to their total lifetimes (Padovani \& Matteucci 1993; Kodama 1997):

$$
\tau_{*}(M)=\left(1.2\left(M / M_{\odot}\right)^{-1.85}+0.003\right) \mathrm{Gyr} .
$$

This formula is valid for stars more massive than $6.6 M_{\odot}$, (inclusive of our entire mass range for Type II SNe). Maeder \& Meynet (1989) give slightly different formulas for stars less massive than $60 M_{\odot}$, but the differences do not affect the chemical evolution model appreciably.

Stars more massive than $100 M_{\odot}$ do not form in this model. The Type II SN ejecta are mixed homogeneously and instantaneously into the ISM of the entire dSph.

We adopt the Type II SN nucleosynthetic yields of Nomoto et al. (2006). The symbol $\zeta_{j, I I}(M, Z)$ represents the mass in element $j$ ejected from the Type II SN explosion of a star with an initial mass $M$. It is a function of both initial stellar mass and metallicity. Nomoto et al. tabulated the yields for seven initial masses ranging from $13 M_{\odot}$ to $40 M_{\odot}$ and four metallicities from $Z=0$ to $Z=0.02$. The total mass of the ejecta is always less than the birth mass of the star because the star loses some mass during its lifetime and because some mass is locked up forever in an SN remnant.

Nomoto et al. modeled both normal core-collapse SNe and very energetic hypernovae $(\mathrm{HNe})$. The lowest mass $\mathrm{HN}$ they modeled is $20 M_{\odot}$. The fraction of stars at least this massive that explode as $\mathrm{HNe}$ is $\epsilon_{\mathrm{HN}}$. Nomoto et al. adopted $\epsilon_{\mathrm{HN}}=0.5$ for their own model of the solar neighborhood. Romano et al. (2010) explored the cases of $\epsilon_{\mathrm{HN}}=0$ and 1. In our own experimentation, we have found that $\epsilon_{\mathrm{HN}}=0$ produces good matches to the dSph abundance patterns at the lowest values of $[\mathrm{Fe} / \mathrm{H}]$, and we adopt this value for the model. In Section 4.2, we explore the effect of increasing $\epsilon_{\mathrm{HN}}$ on the model.

The following integral gives the instantaneous change in gas mass from the ejecta of Type II SNe $\left(M_{\odot} \mathrm{Gyr}^{-1}\right)$ :

$$
\begin{gathered}
\dot{\xi}_{j, \mathrm{II}}=0.31 M_{\odot}^{0.7} \int_{10 M_{\odot}}^{100 M_{\odot}} \zeta_{j, \mathrm{II}}\left(M, Z\left(t-\tau_{*}(M)\right)\right) \\
\times \dot{\xi}_{*}\left(t-\tau_{*}(M)\right) M^{-2.7} d M .
\end{gathered}
$$

The coefficient $0.31 M_{\odot}^{0.7}$ is the normalization from the IMF. This integral depends on the SN yields $\left(\zeta_{j, \text { II }}\right)$, the recent SFH $\left(\dot{\xi}_{*}\right)$, and the high-mass IMF slope $\left(M^{-2.7}\right)$. In practice, this integral is performed numerically with Newton-Cotes integration over an array of 100 logarithmically spaced masses between $10 M_{\odot}$ and $100 M_{\odot}$. The values of $\zeta_{j, \text { II }}$ and $\dot{\xi}_{*}$ are interpolated onto this array. The metallicity used to look up the appropriate $\mathrm{SN}$ yields is consistent with the metallicity of the gas at the time the exploding star formed. (In other words, at any given time step, the metallicities of the lower mass SNe are less than the metallicities of higher mass $\mathrm{SNe}$ from more recently formed stars.)

The instantaneous Type II SN rate $\left(\mathrm{SN} \mathrm{Gyr}^{-1}\right)$ is given by a related integral:

$$
\dot{N}_{\text {II }}=0.31 M_{\odot}^{0.7} \int_{10 M_{\odot}}^{100 M_{\odot}} \dot{\xi}_{*}\left(t-\tau_{*}(M)\right) M^{-2.7} d M .
$$

This integral is performed over the same array of massive star lifetimes as a function of mass as for Equation (7). The value will be used to determine the mass lost from SN winds (Section 2.6).

\subsection{Type Ia Supernovae}

We adopt the Type Ia SN yields of Iwamoto et al. (1999). The mass of element $j$ ejected per Type Ia SN is $\zeta_{j, \mathrm{Ia}}$. The $\mathrm{SNe}$ explode according to a function that approximates the DTD observed by Maoz et al. (2010; see Figure 1). The following equation describes the adopted DTD:

$$
\Psi_{\mathrm{Ia}}=\left\{\begin{array}{ccc}
0 & & t_{\text {delay }}<0.1 \mathrm{Gyr} \\
(1 & \left.\times 10^{-3} \mathrm{SN} \mathrm{Gyr}^{-1} M_{\odot}^{-1}\right) & \\
\times\left(\frac{t_{\text {delay }}}{\mathrm{Gyr}}\right)^{-1.1} & t_{\text {delay }} \geqslant 0.1 \mathrm{Gyr}
\end{array} .\right.
$$

The variable $t_{\text {delay }}$ is used instead of $t$ to indicate that the DTD will be integrated from time $t$ into the past.

Unfortunately, the abundance distributions derived from the chemical evolution model depend sensitively on the normalization and turn-on time of $\Psi_{\text {Ia }}$. Both of these quantities-particularly the turn-on time-have large uncertainties. The normalization affects $[\mathrm{Fe} / \mathrm{H}]$ and the slope of $[\alpha / \mathrm{Fe}]$ with $[\mathrm{Fe} / \mathrm{H}]$. We 


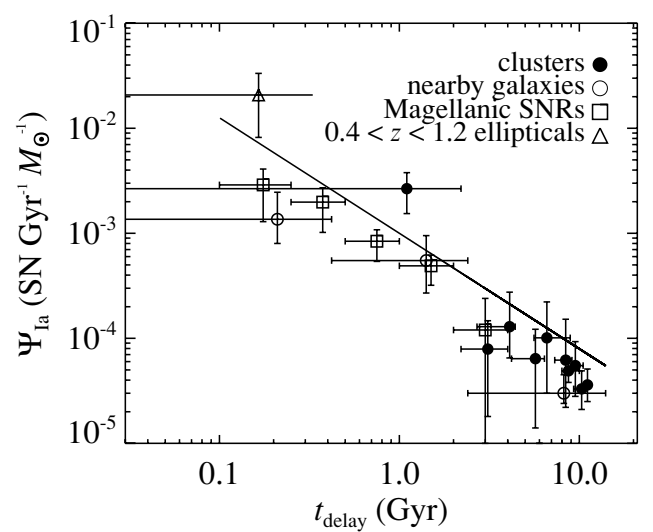

Figure 1. Type Ia SN DTD, as measured by Maoz et al. (2010). The data come from a variety of star formation environments, given in the figure legend. Equation (9) gives the expression for this function. Compare this figure to $\mathrm{MaOz}$ et al.'s Figure 2.

have chosen $1 \times 10^{-3} \mathrm{SN} \mathrm{Gyr}^{-1} M_{\odot}^{-1}$ for the normalization because that is the value that Maoz et al. (2010) reported. Even though the data in Figure 1 are easily consistent with half that value, the larger value better reproduces the slope of $[\alpha / \mathrm{Fe}]$ with $[\mathrm{Fe} / \mathrm{H}]$ for many of the dSphs. The turn-on time determines the time or $[\mathrm{Fe} / \mathrm{H}]$ at which $[\alpha / \mathrm{Fe}]$ begins to drop. We have chosen 0.1 Gyr because that is approximately the maximum value acceptable for the DTD data (Figure 1). See Section 4.1 for a discussion of the effect of increasing this minimum delay time to $0.3 \mathrm{Gyr}$.

The instantaneous Type Ia SN rate is given by combining $\Psi_{\text {Ia }}$ with the past SFH:

$$
\dot{N}_{\text {Ia }}=\int_{t}^{0} \dot{\xi}_{*}\left(t_{\text {delay }}\right) \Psi_{\text {Ia }}\left(t-t_{\text {delay }}\right) d t_{\text {delay }} .
$$

The mass returned to the ISM is the product of the SN Ia yields $\left(\zeta_{j, \text { Ia }}\right)$ and the SN Ia rate:

$$
\dot{\xi}_{j, \text { Ia }}=\zeta_{j, \text { Ia }} \dot{N}_{\text {Ia }}
$$

\subsection{Asymptotic Giant Branch Stars}

Winds from low- and intermediate-mass stars return a small but significant amount of mass to the ISM. The stars lose less than $1 \%$ of this mass before reaching the asymptotic giant branch (AGB; van den Hoek \& Groenewegen 1997). Therefore, we consider mass loss on the AGB only.

We adopt the AGB yields of Karakas (2010), who tracked all of the elements we consider here except $\mathrm{Ca}$ and Ti. (We assume that the fraction of $\mathrm{Ca}$ and $\mathrm{Ti}$ in $\mathrm{AGB}$ ejecta is the same as in the material that formed the star.) We assume all of the mass is ejected in the final time step of the star's lifetime. This assumption is appropriate because an AGB star's thermal pulsation period, during which it loses most of its mass, lasts on the order of 1 Myr (Marigo \& Girardi 2007), which is the length of one time step in our model. Equation (6) gives the lifetimes of stars more massive than $6.6 M_{\odot}$. Less massive stars obey Padovani \& Matteucci's (1993) and Kodama's (1997) equation:

$$
\tau_{*}(M)=10^{\frac{0.334-\sqrt{1.790-0.2232\left[7.764-\log \left(M / M_{\odot}\right)\right]}}{0.1116}} \mathrm{Gyr} .
$$

Each AGB star ejects $\zeta_{j \text {, AGB }}$ solar masses of element $j$. Stars lighter than $10 M_{\odot}$ participate in AGB mass loss whereas stars heavier than $10 M_{\odot}$ explode as Type II SNe (Section 2.2). The lower mass limit we consider for AGB stars is $0.865 M_{\odot}$, which is the stellar lifetime corresponding to the age of the universe, 13.6 Gyr, according to Equation (12). The AGB mass return rate in $M_{\odot} \mathrm{Gyr}^{-1}$ is given by

$$
\begin{gathered}
\dot{\xi}_{j, \mathrm{AGB}}=0.31 M_{\odot}^{0.2} \int_{0.865 M_{\odot}}^{1 M_{\odot}} \zeta_{j, \mathrm{AGB}}\left(M, Z\left(t-\tau_{*}(M)\right)\right) \\
\times \dot{\xi}_{*}\left(t-\tau_{*}(M)\right) M^{-2.2} d M \\
+0.31 M_{\odot}^{0.7} \int_{1 M_{\odot}}^{10 M_{\odot}} \zeta_{j, \mathrm{AGB}}\left(M, Z\left(t-\tau_{*}(M)\right)\right) \\
\times \dot{\xi}_{*}\left(t-\tau_{*}(M)\right) M^{-2.7} d M .
\end{gathered}
$$

Compared to SN ejecta, AGB ejecta affect the chemical evolution of the elements considered here to a small degree. AGB ejecta are more important for other elements, such as C, $\mathrm{N}$, and $\mathrm{O}$.

\subsection{Gas Infall}

Infall of gas during the star formation lifetime of a dSph is required to explain its MDF (Kirby et al. 2010a, hereafter Paper III). Therefore, our model allows pristine gas to fall into the $\mathrm{dSph}$. The gas has a helium fraction of $Y=X_{\mathrm{He}}(0)=0.2486$, which is the value obtained when the WMAP7 (Larson et al. 2010) baryon-to-photon ratio is applied to the formula of Steigman (2007). The rest of the infalling gas is hydrogen.

The MDFs of the dSphs are generally more peaked than a closed box model predicts. One scenario that explain such a distribution is gas infall that first increases and then decreases (Lynden-Bell 1975; Pagel 1997). We find that a quick increase of the rate of gas falling into the galaxy followed by a slower decrease in the infall rate does well at reproducing the data. We parametrize the gas infall rate as follows:

$$
\dot{\xi}_{j, \text { in }}=A_{\text {in }} X_{j}(t=0)\left(\frac{t}{\mathrm{Gyr}}\right) e^{-t / \tau_{\text {in }}} .
$$

The term $X_{j}(t=0)$ means that the infalling gas is primordial (metal-free). The variables $A_{\text {in }}$ and $\tau_{\text {in }}$ are free parameters in the model.

\subsection{Supernova Winds}

The MDFs of dSphs require gas outflow. If that were not the case, the metallicities would approach the SN yields, which are much larger than observed in even the most metal-rich star in any dSph. Gas may be lost through SN winds, stellar winds, or gas stripping from an external source. All of these sources undoubtedly occur over a dSph's lifetime, but SN winds are the most straightforward to include in a chemical evolution model. We ignore other sources of gas loss.

Our computation of gas loss is fairly simple. The galaxy loses a fixed amount of gas for every SN that explodes. The blownout gas mass does not vary with SN type because the explosion energies for Types II and Ia SNe are similar. See Recchi et al. (2001), Romano et al. (2006), and Marcolini et al. (2008) for 
examples of chemical evolution models that treated the energy input from the two SNe types differently. The rate of gas loss is

$$
\dot{\xi}_{j, \text { out }}=A_{\text {out }} X_{j}\left(\dot{N}_{\text {II }}+\dot{N}_{\text {Ia }}\right) \text {. }
$$

The parameter $A_{\text {out }}$ is a free parameter in the model. An energy argument shows that the ejected gas mass is of the order of $10^{4} M_{\odot} \mathrm{SN}^{-1}$. One SN explodes with a typical energy of $10^{51} \mathrm{erg}$ (Woosley \& Weaver 1995). In the late stages of expansion, the kinetic energy of the ejecta is $E_{\text {ej }} \sim 8.5 \times 10^{49}$ erg (Thornton et al. 1998). A typical line-of-sight velocity dispersion for a dwarf galaxy is $\sigma_{\text {los }} \sim 10 \mathrm{~km} \mathrm{~s}^{-1}$. Given the virial theorem $\left(G M / R=3 \sigma_{\text {los }}^{2}\right)$ and the escape velocity $\left(v_{\mathrm{esc}}^{2}=2 G M / R\right)$, the gas mass ejected as a result of SN blowout is $M_{\mathrm{ej}}=E_{\mathrm{ej}} / v_{\mathrm{esc}}^{2}=$ $E_{\mathrm{ej}} /\left(6 \sigma_{\text {los }}^{2}\right) \sim 7 \times 10^{3} M_{\odot} \mathrm{SN}^{-1}$.

A metal-enhanced wind can prevent the galaxy from becoming too metal-rich without such a large gas loss (Vader 1986). For simplicity, we assume that the SN winds have the same chemical content as the gas remaining in the galaxy. See Section 4.3 for a further discussion of including metal-enhanced winds in the model.

\subsection{Complete Chemical Evolution Equation}

The complete equation that describes the chemical evolution of the galaxy's gas is

$$
\begin{aligned}
\xi_{j}(t)= & M_{\mathrm{gas}}(0)+\int_{0}^{t}\left(-\dot{\xi}_{j, *}+\dot{\xi}_{j, \mathrm{II}}+\dot{\xi}_{j, \mathrm{Ia}}\right. \\
& \left.+\dot{\xi}_{j, \mathrm{AGB}}+\dot{\xi}_{j, \mathrm{in}}-\dot{\xi}_{j, \text { out }}\right) d t .
\end{aligned}
$$

The initial gas mass, $M_{\text {gas }}(0)$, is a free parameter. A non-zero initial gas mass may seem inconsistent with Equation (5) because the gas should form stars as it falls into the galaxy. However, the galaxy could acquire gas available for star formation-via gravitational collapse or cooling, for example-on a timescale faster than the star formation (SF) timescale. We will show that the non-zero initial gas mass is more important for the more luminous dSphs.

\subsection{Shortcomings of the Model}

Our model incorporates realistic conditions in dwarf galaxies. We model chemical evolution using an observed Type Ia SN DTD (Maoz et al. 2010). We also take into account the lifetimes of Type II SN progenitors, rather than assuming instantaneous recycling. The delay helps to shape the metal-poor abundance distributions because it affects the rapid rise in metallicity after the onset of star formation.

However, our model is not as sophisticated as some other chemical evolution models of dwarf galaxies (e.g., Marcolini et al. 2008; Revaz et al. 2009; Sawala et al. 2010). In the next section, we show the best model fits to eight different MW satellite galaxies. The simplicity of our model reduces the computational demand of finding the best solution. Nonetheless, we enumerate some shortcomings which affect the interpretation of the abundance distributions.

1. The turn-on time for Type Ia SNe is poorly constrained. Maoz et al. (2010) showed that it is almost certainly 0.1 Gyr or less (at least in the Magellanic Clouds and higher redshift elliptical galaxies), but the DTD slope $\left(t_{\text {delay }}^{-1.1}\right)$ is divergent as $t_{\text {delay }}$ approaches zero. Therefore, the number of Type Ia SNe that explode shortly after their progenitors form depends sensitively on the turn-on time. The uncertainty in the turn-on time translates to a large uncertainty in the Fe abundance distribution. With all other model parameters held fixed, an earlier turn-on time would cause the metallicity of the MDF peak to increase and $[\alpha / \mathrm{Fe}]$ at a given metallicity to decrease. See Matteucci et al. (2009) for a detailed discussion of the effect of adjusting the ratio of prompt to delayed Type Ia SNe.

2. The SN yields are imperfect. As we mention in Section 3, we needed to increase the $[\mathrm{Mg} / \mathrm{H}]$ output of the model by 0.2 dex (see François et al. 2004). Furthermore, Ti is severely underproduced in our model. Therefore, we do not consider Ti abundances at all.

3. Our model assumes instantaneous mixing. Relaxing this approximation would require multiple zones, which we do not consider for the sake of computational simplicity. See Mori et al. (2002), Marcolini et al. (2006, 2008), Revaz et al. (2009), and Sawala et al. (2010) for three-dimensional chemical models of dwarf galaxies.

4. We also assume instantaneous gas cooling. The cooling time for gas to become available for star formation (after accretion or ejection from $\mathrm{SNe}$ and AGB stars) may be longer than the model time step, $\Delta t=1 \mathrm{Myr}$. A more proper treatment of the cooling time, such as in a hydrodynamical model, might result in slightly longer SF durations that we derive with instantaneous cooling.

5. On a related note, we also ignore dynamical processes. Our adoption of a single value of $A_{\text {out }}$, the gas ejected from the galaxy in the wind of one $\mathrm{SN}$, implicitly assumes that the potential of the galaxy is homogeneous and static. This assumption is inconsistent with our allowance of gas to flow into the galaxy. Although dark matter dominates the dynamical mass of dSphs, they undoubtedly change their dark matter masses during their star formation lifetimes (Robertson et al. 2005; Bullock \& Johnston 2005; Johnston et al. 2008). Furthermore, baryonic (adiabatic) contraction can affect star formation and feedback in the dense centers of the dSphs (Napolitano et al. 2010).

6. We consider only one parametrization of the gas infall rate. Because the SFR is proportional to the gas mass, the gas infall rate essentially shapes the differential MDF. Differently shaped gas infall histories might better reproduce the $\mathrm{dSph}$ MDFs. External influences on the gas flow (or alternatively, availability of gas cool enough to form stars) that we do not consider include reionization (Bullock et al. 2000) and tidal and ram pressure stripping (Lin \& Faber 1983).

7. We model only one episode of star formation. CMDs have revealed extended and possibly bursty SFHs in several dSphs in our sample (Fornax and Leo I and II). These bursts will not be included in our model. In these cases, we defer to the photometrically derived SFHs. In fact, we suggest for future study a more sophisticated analysis that models both the CMD and abundance distributions.

8. The infalling gas is assumed to be metal-free at all times. In reality, the metallicity may have increased over time because the source of the new gas may have been blowout from prior SF episodes in the galaxy in question or other galaxies. This gas would have been enriched by $\mathrm{SNe}$ and other nucleosynthetic sources.

9. The modeling result for a given galaxy represents only part of that galaxy's stellar population. Our spectroscopic samples were centrally concentrated to maximize the number of member stars on a DEIMOS slitmask, but most dSphs have 
radial population gradients (e.g., Sculptor, Battaglia et al. 2008). As a result, we preferentially probe the younger, more metal-rich populations. MW satellite galaxies also shed stars as they interact with the Galaxy. Majewski et al. (2000b) identified stars from the Carina dSph beyond Carina's tidal radius. Majewski et al. (2002) and Muñoz et al. (2006) discussed the implications for Carina's present stellar population. In particular, the remaining stars are on average younger and more metal-rich than the lost stars. Consequently, the spectroscopic sample favors the younger, more metal-rich stars.

Some of these shortcomings are observational or theoretical uncertainties (1-2), which can only be resolved with a more thorough investigation of SN rates or yields. Others are simplifications (3-8), which can be resolved with more sophisticated models. The last shortcoming (9) could be resolved by an intensive, wide-field campaign with the intent to recover spectra for a magnitude-limited sample of red giants in a dSph. This project would require a great deal of telescope time, but it could be accomplished in principle for one or two dSphs. Foreground contamination could be minimized by selecting a dSph at high Galactic latitude or photometrically pre-selecting likely members (e.g., Majewski et al. 2000a).

\section{GAS FLOW AND STAR FORMATION HISTORIES}

We apply our chemical evolution model to eight dSphs: Fornax, Leo I, Sculptor, Leo II, Sextans, Draco, Canes Venatici I, and Ursa Minor. We use the abundance measurements from Paper II. For each galaxy, we attempt to match simultaneously the distribution of $[\mathrm{Fe} / \mathrm{H}]$ and the trends of $[\mathrm{Mg} / \mathrm{Fe}],[\mathrm{Si} / \mathrm{Fe}]$, and $[\mathrm{Ca} / \mathrm{Fe}]$ with $[\mathrm{Fe} / \mathrm{H}]$ by adjusting the six free parameters listed at the bottom of Table 1 .

Unfortunately, some elemental abundances could not be matched for any combination of parameter values. In particular, the model underpredicts $[\mathrm{Mg} / \mathrm{H}]$ and $[\mathrm{Ti} / \mathrm{H}]$. François et al. (2004) constructed a chemical evolution model for the MW and also encountered trouble in reproducing the yields. They concluded that the SN yields should be modified. They specifically singled out $\mathrm{Mg}$ for being underproduced by both Type Ia SNe and low-mass Type II SNe. We feel comfortable modifying the model results for $[\mathrm{Mg} / \mathrm{H}]$ because chemical evolution models by different authors over a wide range of galaxy masses and ages indicate that such modification is necessary. We add 0.2 dex to $[\mathrm{Mg} / \mathrm{H}]$ to bring the model into better agreement with the data. However, the Nomoto et al. (2006) Type II SN yield for [Ti/Fe] is about -0.1 dex, which is far below the value observed for metal-poor stars in dSphs or in the MW halo. Rather than attempting to correct such a large deficit, we ignore the model result for Ti. Nomoto et al. also ignore their Ti yields in their own chemical evolution model of the solar neighborhood.

In Paper III, we found the best-fit analytical chemical evolution models for the same eight $\mathrm{dSphs}$ based on their MDFs alone. We repeat the process here for our more sophisticated model. As in Paper III, we use maximum likelihood estimation to find the best-fit model parameters.

The likelihood that a particular model matches the data is the product of probability distributions. Each star is represented by a probability distribution in a four-dimensional space. The four dimensions are $[\mathrm{Fe} / \mathrm{H}],[\mathrm{Mg} / \mathrm{Fe}],[\mathrm{Si} / \mathrm{Fe}]$, and $[\mathrm{Ca} / \mathrm{Fe}]$. We denote these quantities as $\epsilon_{i, j}$, where $i$ represents the $i^{\text {th }}$ star and $j$ identifies one of the four element ratios. The Gaussian is centered on the star's observed values. The width in each axis is the estimate of measurement uncertainty $\left(\delta \epsilon_{i, j}\right)$ in that quantity. Stars with larger uncertainties have less weight in the likelihood calculation than stars with smaller uncertainties. (Although Figures 2-9 show only stars with uncertainties less than 0.3 dex, there is no error cut in the likelihood calculation. Instead, we downweight stars with large uncertainties.) The chemical evolution model traces a path $\epsilon_{j}(t)$ in the four-dimensional space. The probability that a star formed at a point $t$ on the path is $d P / d t=\dot{M}_{*}(t) / M_{*}$, where $M_{*}$ is the galaxy's final stellar mass. The likelihood that one star conforms to the model is the line integral of $d P / d t$ along the path $\epsilon_{j}(t)$. The total likelihood $L$ is the product of the individual likelihoods of the $N$ stars:

$$
\begin{aligned}
L=\prod_{i=1}^{N} \int_{0}^{t}\left(\prod_{j} \frac{1}{\sqrt{2 \pi} \delta \epsilon_{i, j}} \exp \frac{-\left(\epsilon_{i, j}-\epsilon_{j}(t)\right)^{2}}{2\left(\delta \epsilon_{i, j}\right)^{2}}\right) \frac{\dot{M}_{*}(t)}{M_{*}} d t \\
\quad \times\left(\frac{1}{\sqrt{2 \pi} \delta M_{*, \mathrm{obs}}} \exp \frac{-\left(M_{*, \mathrm{obs}}-M_{*, \mathrm{model}}\right)^{2}}{2\left(\delta M_{*, \mathrm{obs}}\right)^{2}}\right. \\
\left.\quad \times \frac{1}{\sqrt{2 \pi} \delta M_{\mathrm{gas}, \mathrm{obs}}} \exp \frac{-\left(M_{\mathrm{gas}, \mathrm{obs}}\right)^{2}}{2\left(\delta M_{\mathrm{gas}, \mathrm{obs}}\right)^{2}}\right)^{0.1 N} .
\end{aligned}
$$

The second line of the equation requires that the final stellar mass of the model $\left(M_{* \text {, model }}\right)$ matches the observed stellar mass $\left(M_{*, \mathrm{obs}}\right)$ within the observational uncertainties. We adopt the stellar masses of Woo et al. (2008). They did not study Canes Venatici I. We assume that the galaxy has about the same stellar mass as Ursa Minor because it has the same luminosity within the observational uncertainties. The third line of the equation ensures that the dSph ends up gas free. We fairly arbitrarily assume an uncertainty of $\delta M_{\text {gas,obs }}=10^{3} M_{\odot}$ because even lower values of $\delta M_{\text {gas,obs }}$ cause the chemical evolution model to converge on spurious solutions. The exponent $0.1 \mathrm{~N}$ sets the relative influence of the final stellar and gas mass compared to the abundance distributions. This value was chosen so that these quantities did not dominate the likelihood but also so that the modeled galaxies ended up gas-free and with about the correct stellar mass.

For computational simplicity, we minimize the quantity $\hat{L}=-\ln L$ :

$$
\begin{aligned}
\hat{L}= & -\sum_{i=1}^{N} \ln \int_{0}^{t}\left(\prod_{j} \frac{1}{\sqrt{2 \pi} \delta \epsilon_{i, j}^{2}} \exp \frac{-\left(\epsilon_{i, j}-\epsilon_{j}(t)\right)^{2}}{2 \delta \epsilon_{i, j}^{2}}\right) \\
& \times \frac{\dot{M}_{*}(t)}{M_{*}} d t+0.1 N\left(\frac{\left(M_{*, \mathrm{obs}}-M_{*, \text { model }}\right)^{2}}{2\left(\delta M_{*, \mathrm{obs}}\right)^{2}}\right.
\end{aligned}
$$$$
\left.+\frac{\left(M_{\mathrm{gas}, \mathrm{obs}}\right)^{2}}{2\left(\delta M_{\mathrm{gas}, \mathrm{obs}}\right)^{2}}+\ln (2 \pi)+\ln \left(\delta M_{*, \mathrm{obs}}\right)+\ln \left(\delta M_{\mathrm{gas}, \mathrm{obs}}\right)\right) .
$$

We find the values of the six parameters that minimize $\hat{L}$ using Powell's method. We calculate uncertainties on the model parameters via a Monte Carlo Markov chain. We perform at least $10^{4}$ trials for each $\mathrm{dSph}$ after a burn-in period of $10^{3}$ trials. The dSphs with shorter SF durations require less computation time, and we were able to perform up to $5 \times 10^{4}$ trials for some of the dSphs. As in Paper III, the model uncertainties are 

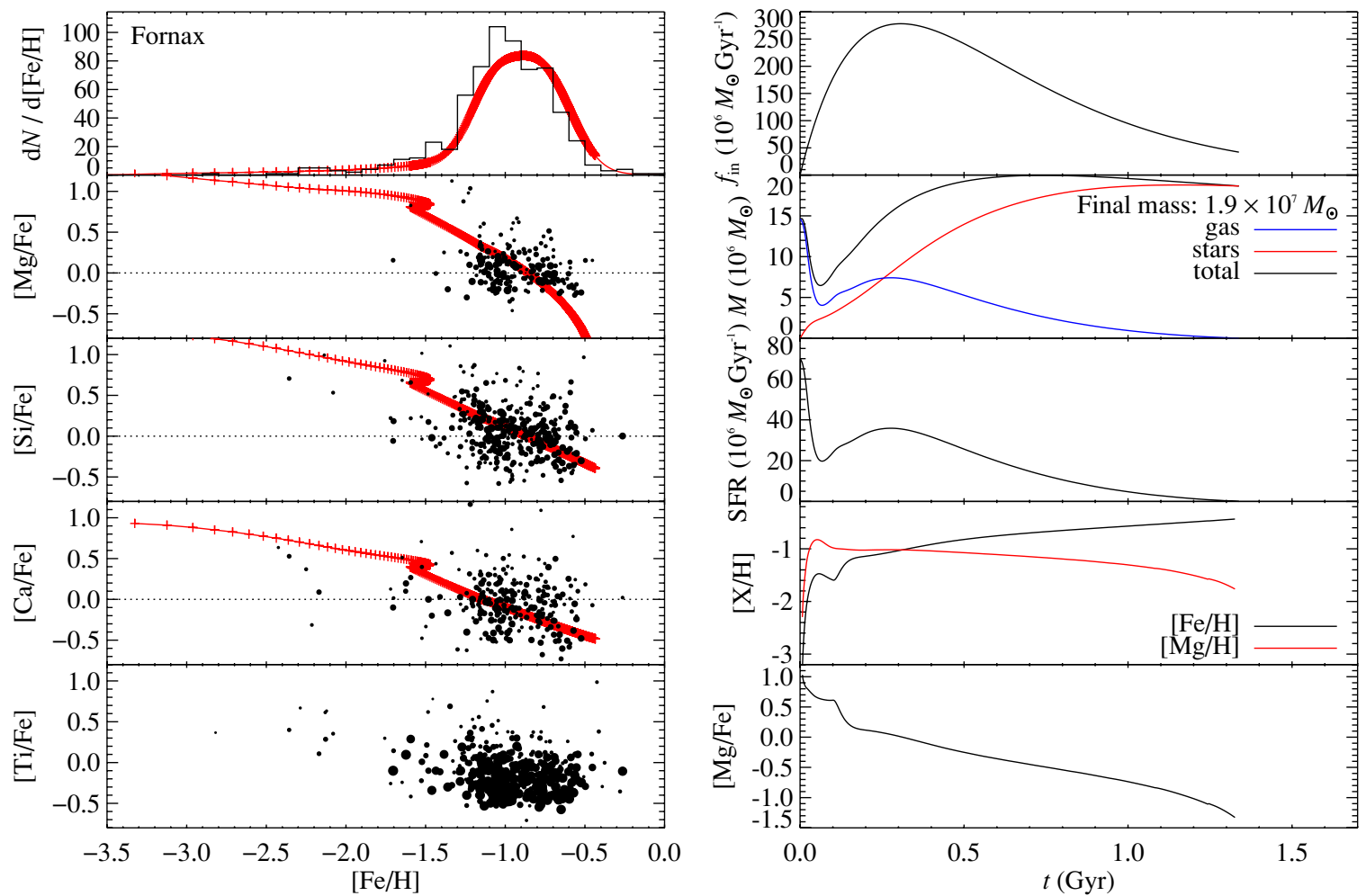

Figure 2. Observed abundance ratios and the best-fit gas flow and SFH model for Fornax. Left: the top panel shows the observed MDF as the black histogram and the modeled MDF in red. The model is convolved with an uncertainty function to mimic the broadening of the histogram induced by observational error. A cross marks each $1 \mathrm{Myr}$ time step, but these are too closely spaced to discern for most of the metallicity range. Very few stars are expected to have formed at the low metallicities where the crosses are distinguishable. The other panels show the observed $[\mathrm{Mg} / \mathrm{Fe}],[\mathrm{Si} / \mathrm{Fe}],[\mathrm{Ca} / \mathrm{Fe}]$, and $[\mathrm{Ti} / \mathrm{Fe}]$ ratios as black points whose sizes are inversely proportional to measurement uncertainties. Only points with uncertainties less than 0.3 dex are shown. The red lines show the abundance ratios of the stars and gas at each time step. We do not show the model results for [Ti/Fe] because the SN yields are inaccurate. Right: the gas flow and SFH for the best-fit model. From top to bottom, the panels show the gas inflow rate; the stellar, gas-phase, and total baryonic mass; the SFR; the iron and magnesium abundances; and the [Mg/Fe] ratio, all as a function of time. The second panel also gives the final stellar mass in the model.

(A color version of this figure is available in the online journal.)
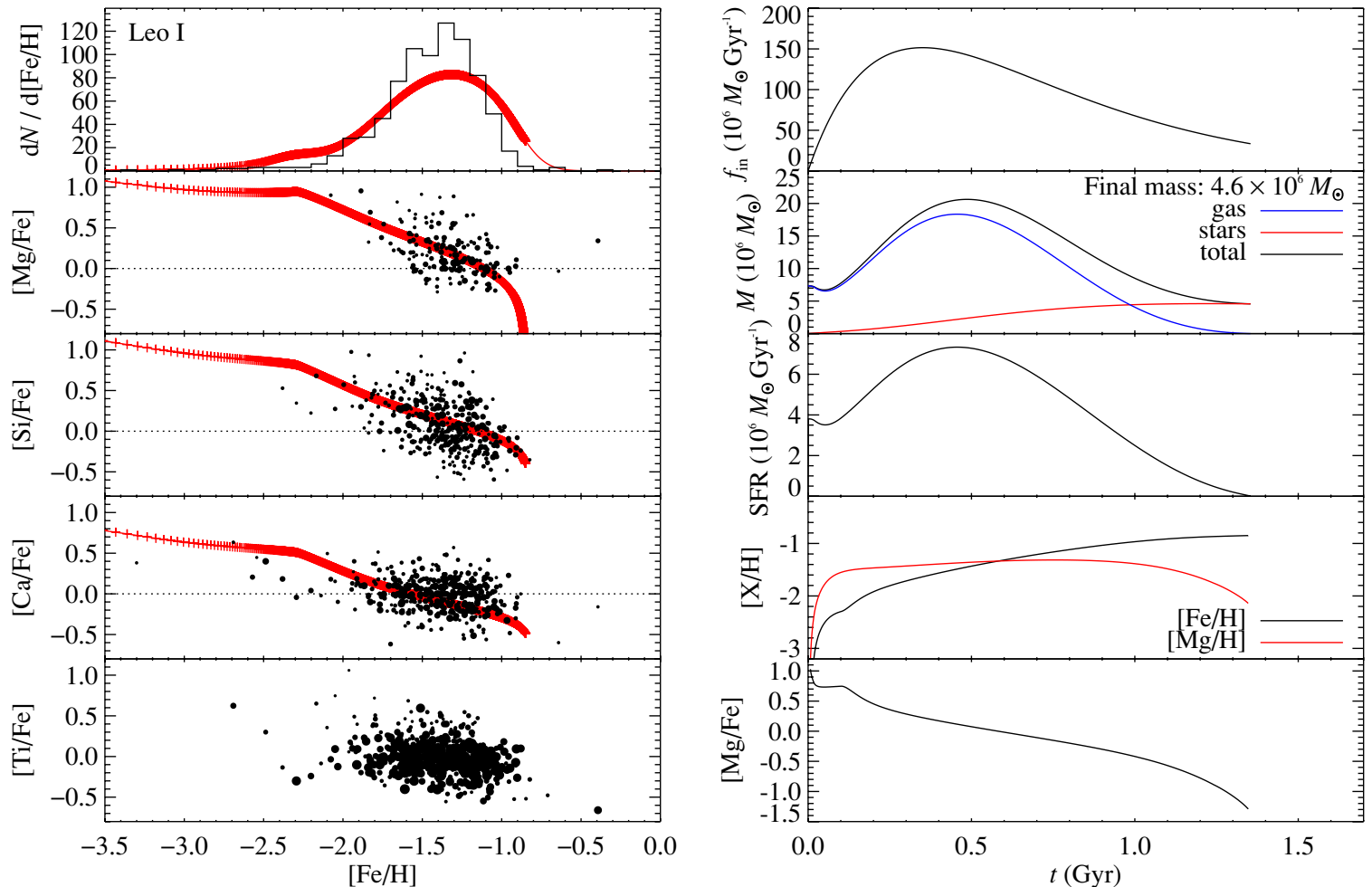

Figure 3. Observed abundance ratios and the best-fit gas flow and SFH model for Leo I. See Figure 2 for a detailed explanation.

(A color version of this figure is available in the online journal.) 

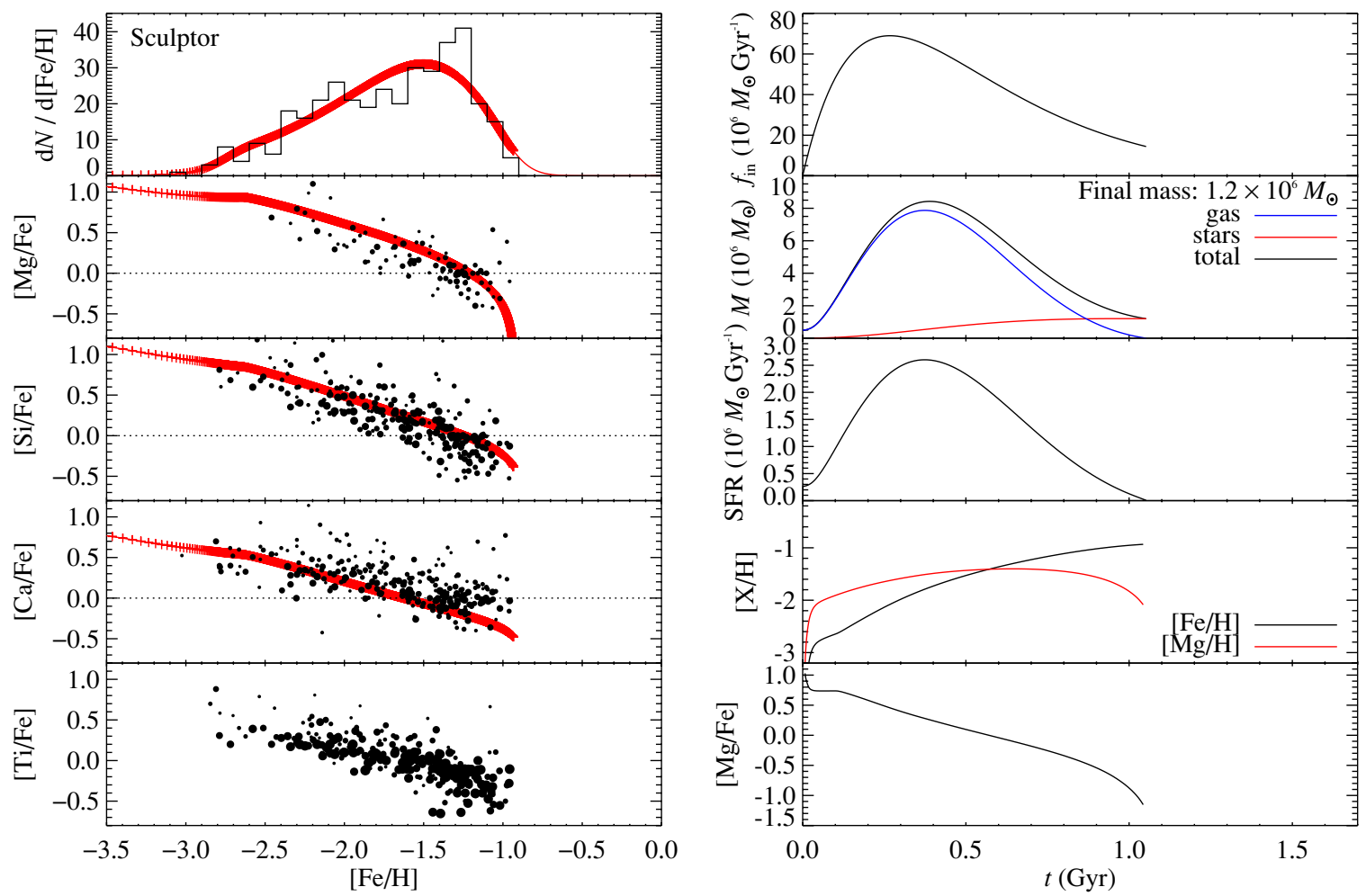

Figure 4. Observed abundance ratios and the best-fit gas flow and SFH model for Sculptor. See Figure 2 for a detailed explanation.

(A color version of this figure is available in the online journal.)
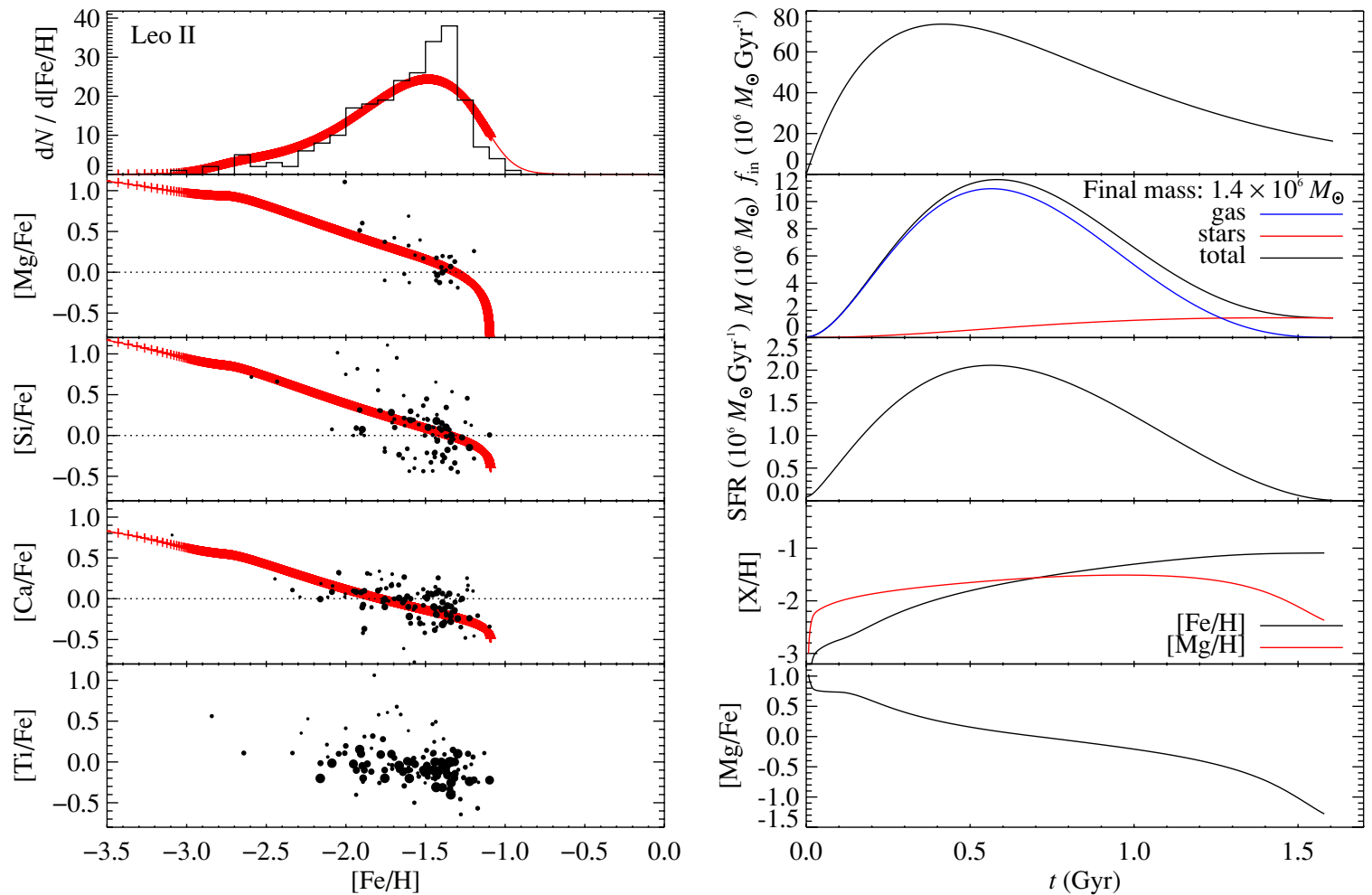

Figure 5. Observed abundance ratios and the best-fit gas flow and SFH model for Leo II. See Figure 2 for a detailed explanation.

(A color version of this figure is available in the online journal.)

the two-sided $68.3 \%$ confidence intervals. These uncertainties incorporate only observational uncertainty and not systematic model errors. Table 2 lists the solutions for each $\mathrm{dSph}$ in order of decreasing luminosity.
Table 3 lists the total star formation durations for the most likely models. The duration is not a free parameter but a result of the model. The table also lists some timescales derived from HST CMDs (Dolphin et al. 2005; Orban et al. 2008). It is not 

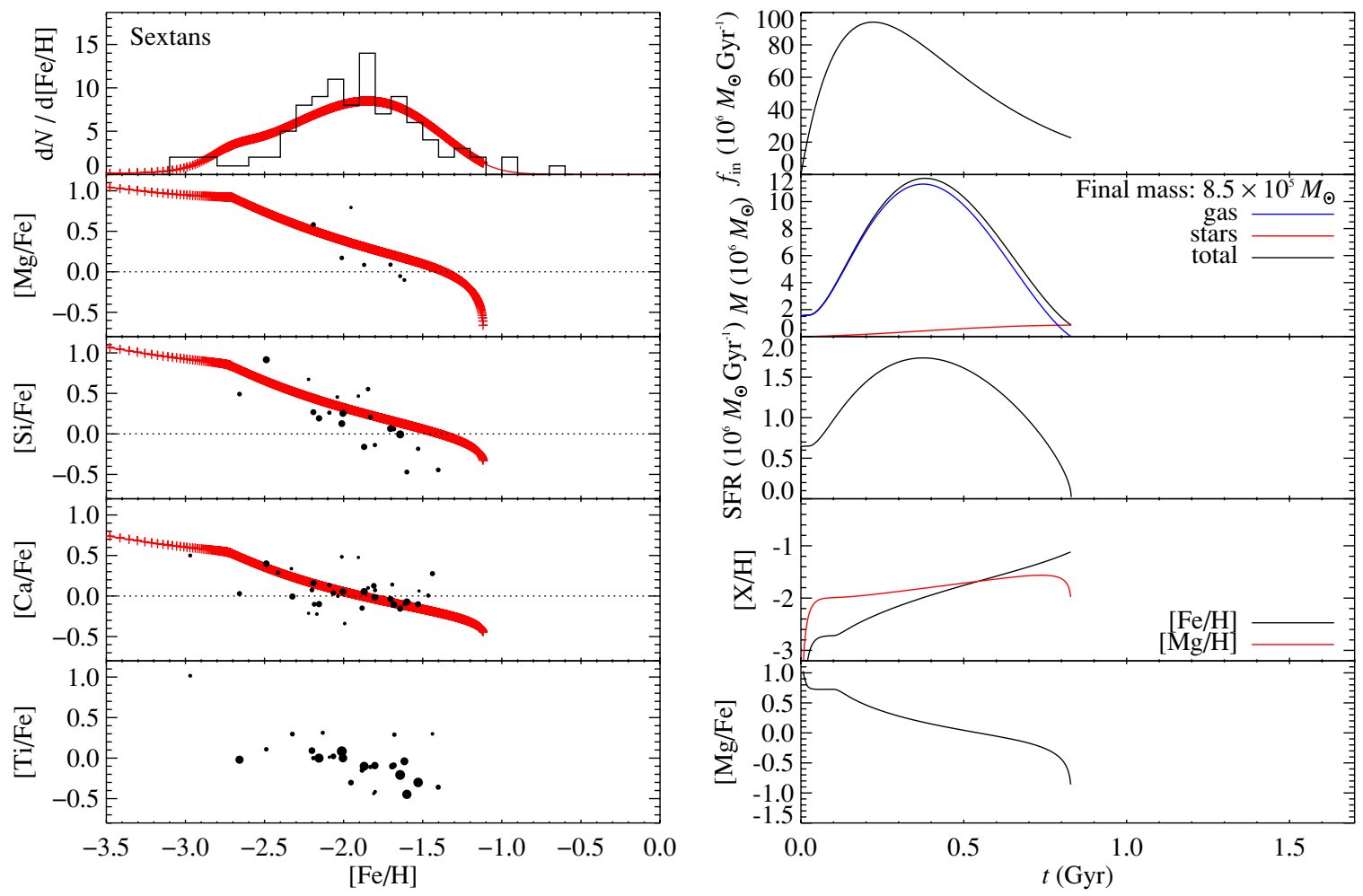

Figure 6. Observed abundance ratios and the best-fit gas flow and SFH model for Sextans. See Figure 2 for a detailed explanation.

(A color version of this figure is available in the online journal.)
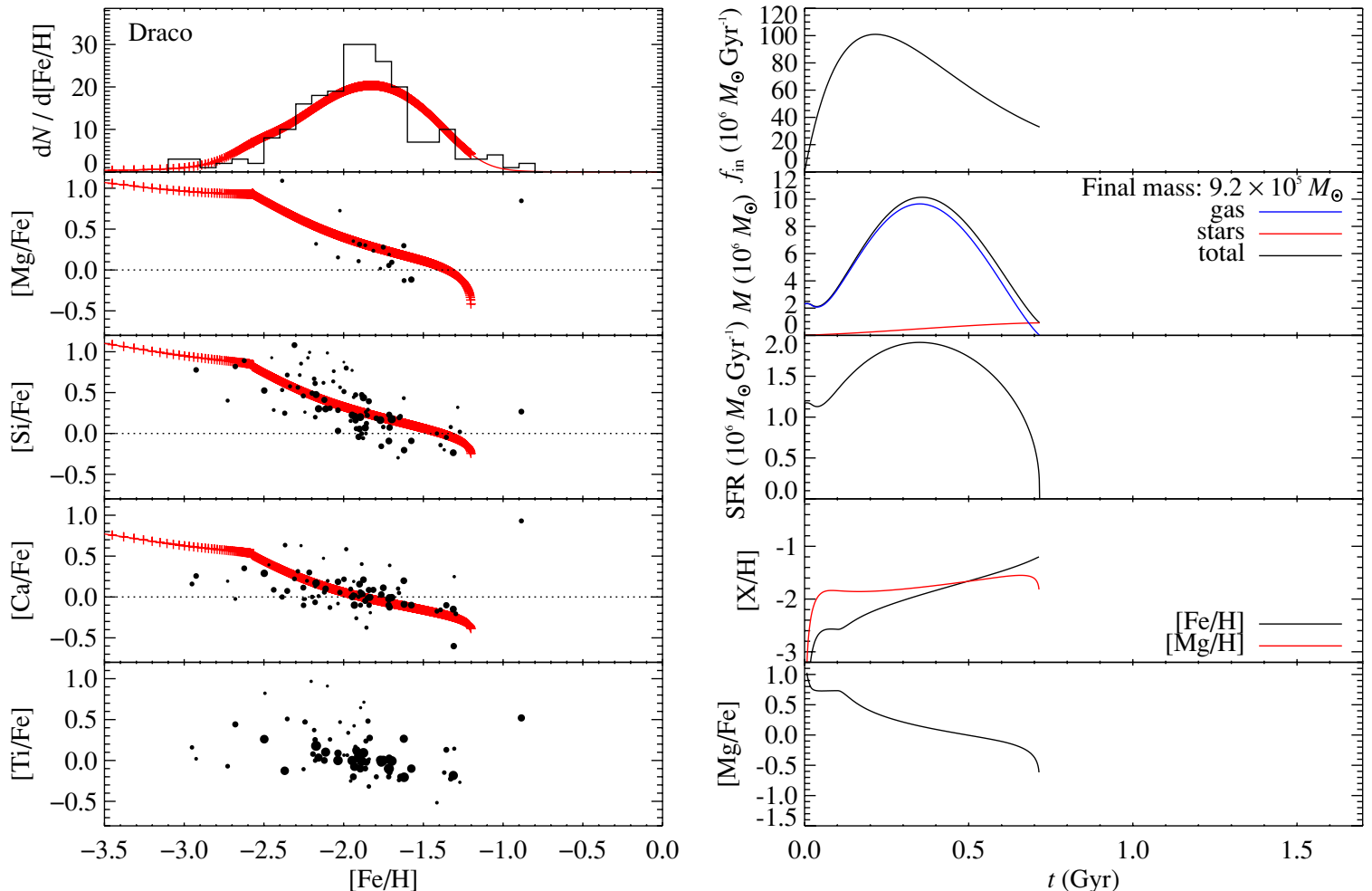

Figure 7. Observed abundance ratios and the best-fit gas flow and SFH model for Draco. See Figure 2 for a detailed explanation. (A color version of this figure is available in the online journal.)

possible to measure photometrically the total star formation duration for predominantly ancient stellar populations because $10 \mathrm{Gyr}$ isochrones are extremely similar to $13 \mathrm{Gyr}$ isochrones. Therefore, we have quoted $f_{10 \mathrm{G}}$, the fraction of stars formed more recently than $10 \mathrm{Gyr}$. For small or zero values of $f_{10 \mathrm{G}}$, the CMD shows that the population is ancient, but there is no time resolution. We also show the stellar mass-weighted mean age $\tau$ (Orban et al. 2008). For the three dSphs with intermediate-aged 

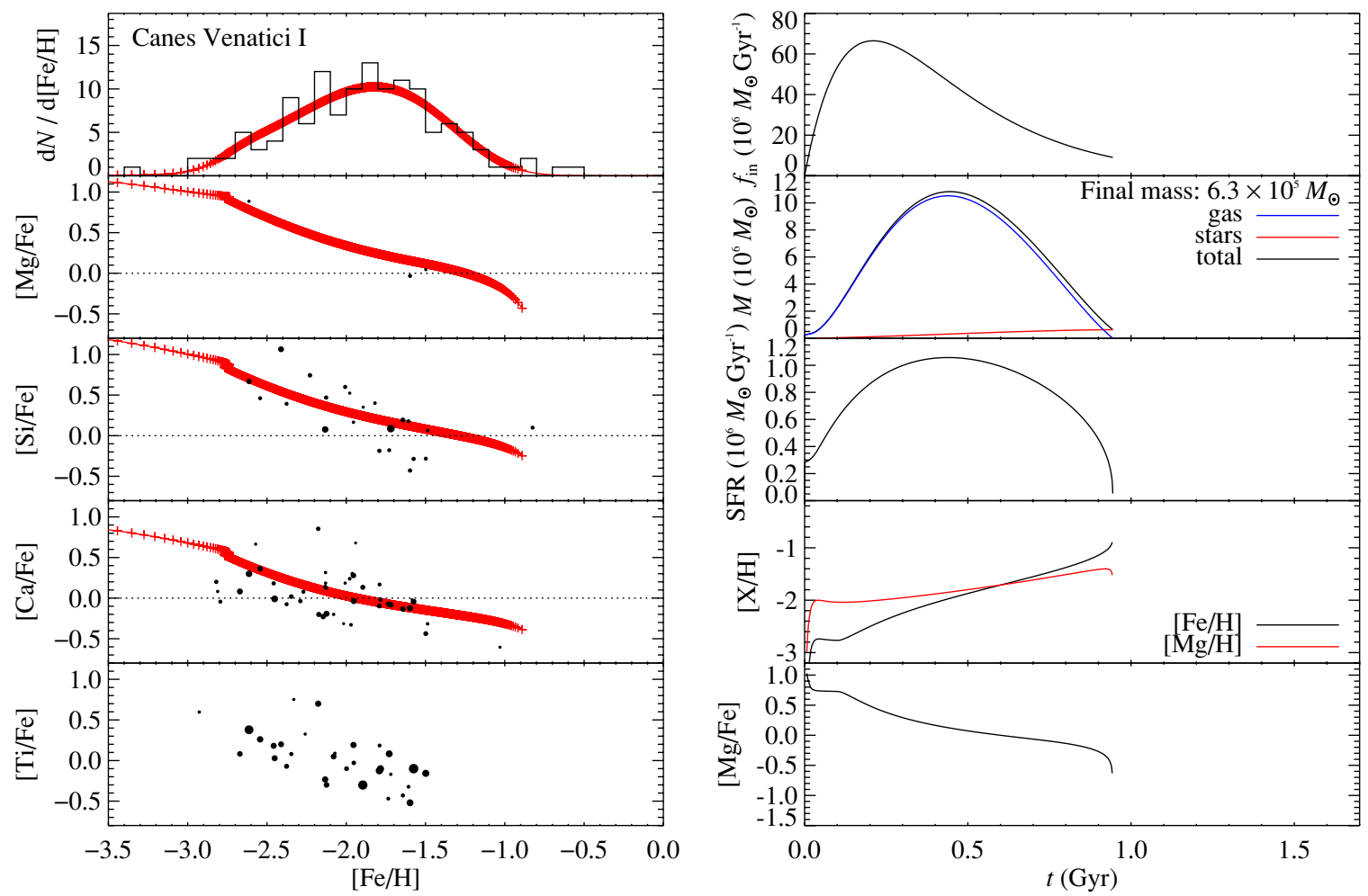

Figure 8. Observed abundance ratios and the best-fit gas flow and SFH model for Canes Venatici I. See Figure 2 for a detailed explanation.

(A color version of this figure is available in the online journal.)
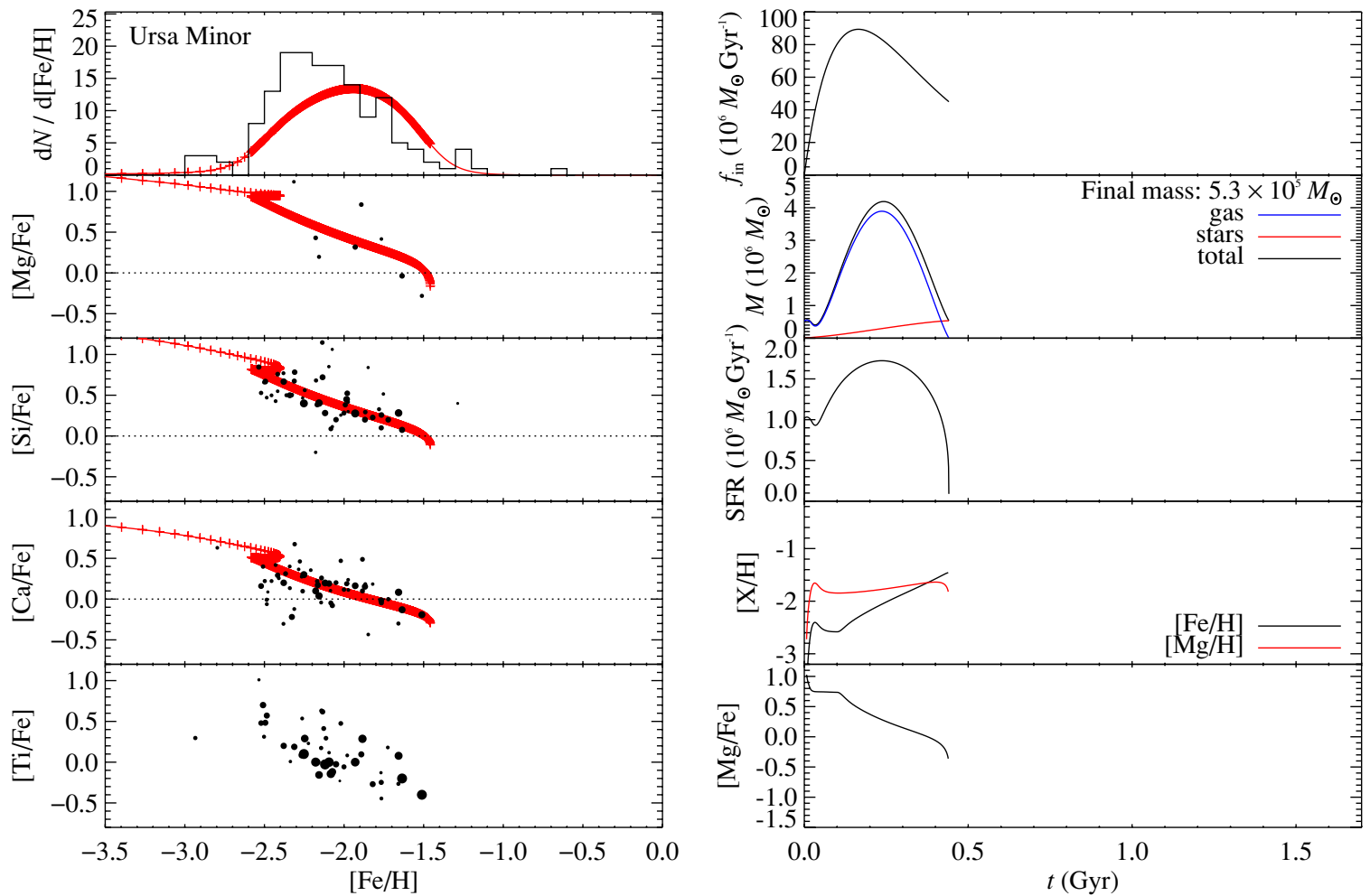

Figure 9. Observed abundance ratios and the best-fit gas flow and SFH model for Ursa Minor. See Figure 2 for a detailed explanation.

(A color version of this figure is available in the online journal.)

populations (Fornax and Leo I and II), $\tau$ combined with $f_{10 \mathrm{G}}$ gives some idea of the star formation duration. For example, Fornax formed $1-f_{10 \mathrm{G}}=27 \%$ of its stars beyond $10 \mathrm{Gyr}$ ago, but the mean age is just 7.4 Gyr. Half of Fornax's stars formed over at least $2.6 \mathrm{Gyr}$, and the other half formed even more recently. Our abundance-derived duration of $1.3 \mathrm{Gyr}$ is inconsistent with this photometric star formation duration. For Fornax and Leo I and II, we defer to the photometrically derived 
Table 2

Galaxy Properties and Chemical Evolution Model Parameters

\begin{tabular}{|c|c|c|c|c|c|c|c|c|c|c|c|}
\hline dSph & $\begin{array}{c}L \\
\left(10^{5} L_{\odot}\right)\end{array}$ & $\begin{array}{c}M_{*} \\
\left(10^{5} M_{\odot}\right) \\
\end{array}$ & $\begin{array}{c}\sigma_{\mathrm{los}} \\
\left(\mathrm{km} \mathrm{s}^{-1}\right)\end{array}$ & $\begin{array}{c}R_{e} \\
(\mathrm{pc})\end{array}$ & $\begin{array}{r}D_{\mathrm{GC}} \\
(\mathrm{kpc})\end{array}$ & $\begin{array}{c}A_{*} \\
\left(\frac{10^{6} M_{\odot}}{\mathrm{Gyr}}\right)\end{array}$ & $\alpha$ & $\begin{array}{c}A_{\text {in }} \\
\left(\frac{10^{9} M_{\odot}}{\mathrm{Gyr}}\right)\end{array}$ & $\begin{array}{c}\tau_{\text {in }} \\
(\mathrm{Gyr})\end{array}$ & $\begin{array}{c}A_{\text {out }} \\
\left(\frac{10^{3} M_{\odot}}{\mathrm{SN}}\right) \\
\end{array}$ & $\begin{array}{c}M_{\mathrm{gas}}(0) \\
\left(10^{6} M_{\odot}\right)\end{array}$ \\
\hline Fornax & $180 \pm 50$ & $190 \pm 50$ & $10.7 \pm 0.2$ & $714 \pm 40$ & $141 \pm 12$ & $5.02_{-1.00}^{+2.21}$ & $0.98_{-0.04}^{+0.15}$ & $2.46_{-0.17}^{+0.70}$ & $0.31_{-0.04}^{+0.01}$ & $1.51_{-0.06}^{+0.03}$ & $14.58_{-2.46}^{+1.77}$ \\
\hline Leo I & $56 \pm 16$ & $45 \pm 13$ & $9.0 \pm 0.4$ & $295 \pm 49$ & $257 \pm 76$ & $0.92_{-0.05}^{+0.67}$ & $0.71_{-0.17}^{+0.01}$ & $1.17_{-0.10}^{+0.05}$ & $0.35_{-0.01}^{+0.02}$ & $3.89_{-0.08}^{+0.16}$ & $7.32_{-0.21}^{+0.27}$ \\
\hline Sculptor & $22 \pm 10$ & $12 \pm 5$ & $9.0 \pm 0.2$ & $282 \pm 41$ & $85 \pm 23$ & $0.47_{-0.12}^{+0.09}$ & $0.83_{-0.08}^{+0.14}$ & $0.70_{-0.08}^{+0.12}$ & $0.27_{-0.02}^{+0.02}$ & $5.36_{-0.17}^{+0.16}$ & $0.50_{-0.25}^{+0.62}$ \\
\hline Leo II & $6.6 \pm 1.9$ & $14 \pm 4$ & $6.6 \pm 0.5$ & $177 \pm 13$ & $221 \pm 50$ & $0.43_{-0.10}^{+0.93}$ & $0.66_{-0.40}^{+0.17}$ & $0.48_{-0.07}^{+0.23}$ & $0.42_{-0.09}^{+0.05}$ & $6.59_{-0.31}^{+0.26}$ & $0.05_{-0.04}^{+3.00}$ \\
\hline Sextans & $4.1 \pm 1.2$ & $8.5 \pm 2.4$ & $7.1 \pm 0.3$ & $768 \pm 47$ & $98 \pm 13$ & $0.52_{-0.18}^{+0.45}$ & $0.50_{-0.25}^{+0.20}$ & $1.15_{-0.20}^{+0.51}$ & $0.22_{-0.04}^{+0.03}$ & $9.60_{-0.72}^{+0.86}$ & $1.55_{-1.20}^{+2.12}$ \\
\hline Draco & $2.7 \pm 0.4$ & $9.1 \pm 1.4$ & $10.1 \pm 0.5$ & $220 \pm 11$ & $92 \pm 29$ & $0.88_{-0.28}^{+0.30}$ & $0.34_{-0.14}^{+0.16}$ & $1.27_{-0.18}^{+0.25}$ & $0.22_{-0.02}^{+0.02}$ & $9.51_{-0.52}^{+0.43}$ & $2.32_{-1.20}^{+1.06}$ \\
\hline Can. Ven. I & $2.3 \pm 0.4$ & $6 \pm 2$ & $7.6 \pm 0.5$ & $546 \pm 36$ & $210 \pm 29$ & $0.46_{-0.26}^{+0.41}$ & $0.36_{-0.32}^{+0.37}$ & $0.86_{-0.22}^{+0.64}$ & $0.21_{-0.06}^{+0.04}$ & $8.83_{-0.70}^{+0.90}$ & $0.27_{-0.26}^{+0.81}$ \\
\hline Ursa Minor & $2.2 \pm 0.7$ & $5.6 \pm 1.7$ & $11.5 \pm 0.6$ & $445 \pm 44$ & $70 \pm 19$ & $1.21_{-0.11}^{+0.53}$ & $0.26_{-0.12}^{+0.07}$ & $1.47_{-0.13}^{+0.64}$ & $0.17_{-0.03}^{+0.02}$ & $11.04_{-0.65}^{+0.71}$ & $0.54_{-0.17}^{+0.71}$ \\
\hline
\end{tabular}

References. $L$ (luminosity): Martin et al. (2008a) for Canes Venatici I, Irwin \& Hatzidimitriou (1995) for the others. $M_{*}$ (stellar mass): Woo et al. (2008), except that we have assumed that Canes Venatici I has about the same $M_{*}$ as Ursa Minor. $\sigma_{\text {los }}$ (line-of-sight velocity dispersion) and $R_{e}$ (two-dimensional projected half-light radius): Wolf et al. (2010) and references therein. $D_{\mathrm{GC}}$ (Galactocentric distance): coordinates from Mateo (1998). See Paper II, Table 1, for the sources of the heliocentric distances.

Table 3

Star Formation Durations

\begin{tabular}{lcccc}
\hline \hline dSph & $\begin{array}{c}\text { Duration } \\
(\mathrm{Gyr})\end{array}$ & $f_{10 \mathrm{G}}(\mathrm{D} 05)^{\mathrm{b}}$ & $f_{10 \mathrm{G}}(\mathrm{O} 08)^{\mathrm{c}}$ & $\begin{array}{c}\tau(\mathrm{O} 08)^{\mathrm{d}} \\
(\mathrm{Gyr})\end{array}$ \\
\hline Fornax & 1.3 & 0.73 & 0.73 & 7.4 \\
Leo I & 1.4 & 0.75 & 0.76 & 6.4 \\
Sculptor & 1.1 & 0.05 & 0.14 & 12.6 \\
Leo II & 1.6 & 0.56 & 0.70 & 8.8 \\
Sextans & 0.8 & 0.00 & $0.00^{\mathrm{e}}$ & 12.0 \\
Draco & 0.7 & 0.06 & 0.49 & 10.9 \\
Can. Ven. I & 0.9 & $\ldots$ & $\ldots$ & $\ldots$ \\
Ursa Minor & 0.4 & 0.00 & $0.00^{\mathrm{e}}$ & 12.0 \\
\hline
\end{tabular}

Notes. Our star formation durations for Fornax and Leo I and II are almost certainly too short because our chemical evolution model does not permit multiple SF bursts.

a Star formation duration derived from our model, based on spectroscopic, multi-element abundances.

${ }^{\mathrm{b}}$ Fraction of stars formed more recently than $10 \mathrm{Gyr}$ ago, based on an analysis of HST photometry (Dolphin et al. 2005).

${ }^{\mathrm{c}}$ Fraction of stars formed more recently than $10 \mathrm{Gyr}$ ago, based on a different analysis of $H S T$ photometry (Orban et al. 2008).

d Stellar mass-weighted mean age, based on HST photometry (Orban et al. 2008).

${ }^{\mathrm{e}}$ Orban et al. (2008) did not measure these values but took them from Dolphin et al. (2005).

SFHs (see item 7 of Section 2.8). They are more realistic because they permit an arbitrary number of SF episodes. For the galaxies whose CMDs identify them to be ancient, our abundance distributions are far more sensitive probes of the SF duration than the CMD.

In the following sections, we discuss the derived star formation and gas flow histories for each $\mathrm{dSph}$ and compare them to previous photometrically and spectroscopically derived SFHs.

\subsection{Fornax}

We begin our discussion with the most luminous of the mostly intact MW dSph satellites, Fornax. Its $[\alpha / \mathrm{Fe}]$ distribution (Figure 2) shows the least evidence of correlation with $[\mathrm{Fe} / \mathrm{H}]$ of all eight dSphs studied here. In the range $-1.3 \lesssim[\mathrm{Fe} / \mathrm{H}] \lesssim$ -0.5 , the four $[\alpha / \mathrm{Fe}]$ element ratios span almost 1 dex at a fixed metallicity with no evidence of a slope with $[\mathrm{Fe} / \mathrm{H}]$. The rarer stars more metal poor than $[\mathrm{Fe} / \mathrm{H}] \approx-1.3$ have higher average $[\alpha / \mathrm{Fe}]$.
The large range of $[\alpha / \mathrm{Fe}]$ and the lack of correlation with $[\mathrm{Fe} / \mathrm{H}]$ each suggest bursty or inhomogeneous star formation. A bursty SFH would cause spikes and depressions in $[\alpha / \mathrm{Fe}]$ as $[\mathrm{Fe} / \mathrm{H}]$ increases monotonically (e.g., Gilmore \& Wyse 1991), even if the star formation were well-mixed over the whole galaxy at all times. Measurement uncertainties might blur the division between the $[\alpha / \mathrm{Fe}]$ spikes in different bursts. Alternatively, if the SN nucleosynthetic products were not well mixed, the $[\alpha / \mathrm{Fe}]$ value of a star would reflect the particular SFH of its birth site rather than the galaxy as a whole. Consequently, the abundance distribution would be a composite of several different SFHs. Coupled with measurement uncertainties, the composite distribution may look like an uncorrelated scatter of points, such as the distribution in Figure 2. Burstiness and inhomogeneity are not mutually exclusive. Both processes might have affected Fornax's SFH.

Based on HST/Wide Field Planetary Camera 2 (WFPC2) photometry, Buonanno et al. (1999) surmised that the field (not GC) population of Fornax endured three major bursts of star formation separated by about 3 Gyr. Saviane et al. (2000), Battaglia et al. (2006), Gullieuszik et al. (2007), and Coleman \& de Jong (2008) provided additional photometric and spectroscopic evidence of multiple discrete populations, including a burst $4 \mathrm{Gyr}$ ago. Grebel \& Stetson (1999), Battaglia et al., and Coleman \& de Jong additionally showed that the younger, more metal-rich populations are more centrally concentrated. Thus, it seems that star formation in Fornax was both bursty and inhomogeneous.

Our chemical evolution model is incompatible with Fornax's complex SFH. First, we model the SFR as a smooth function, not a bursty one. Second, the model has only one zone and does not account for spatially segregated star formation. Consequently, the SFH derived from our model should be viewed with skepticism. Most notably, we derive a total star formation duration of $1.3 \mathrm{Gyr}$ (the time at which star formation and SN winds exhausted the gas supply, thereby truncating star formation), whereas every photometric study shows that star formation in Fornax lasted for most of the age of the universe. In addition, the model does not match the observed flatness of the $[\alpha / \mathrm{Fe}]$ distribution for the bulk of the stars. However, the model does share one important quality with photometrically derived SFHs: The initial metal enrichment is very rapid. The metallicity in our model reaches $[\mathrm{Fe} / \mathrm{H}]=-1$ at $0.3 \mathrm{Gyr}$ after the commencement of star formation. Pont et al. (2004) 
deduced that Fornax reached $[\mathrm{Fe} / \mathrm{H}]=-1$ within a few Gyr. One advantage of a spectroscopically derived $\mathrm{SFH}$ is that it is sensitive to relative ages, whereas a photometrically derived $\mathrm{SFH}$ is sensitive to absolute ages but has poor age resolution for old populations.

Letarte et al. (2010) measured multi-element abundances from higher resolution spectra of 81 Fornax members. We showed in Paper II that our abundance measurements match theirs very well. They pointed out that centrally selected stars in Fornax will preferentially sample the young, metal-rich component. In fact, the most metal-poor star known in Fornax $([\mathrm{Fe} / \mathrm{H}]=-3.66$, Tafelmeyer et al. 2010) is very far (43') from the center of the $\mathrm{dSph}$. The discovery emphasizes that selecting stars in the center of the dSph biases the age and metallicity distribution.

\subsection{Leo I}

Leo $\mathrm{I}$ is the second most massive dwarf galaxy in our sample. The $[\alpha / \mathrm{Fe}]$ distribution of Leo I shows a moderate correlation with $[\mathrm{Fe} / \mathrm{H}]$. In particular, the lower metallicity stars $([\mathrm{Fe} / \mathrm{H}]<-1.5)$ show on average higher $[\alpha / \mathrm{Fe}]$ (except for Ti) than the more metal-rich stars.

Lee et al. (1993) obtained the first CCD-based CMD of Leo I, and they found hints of a young $(3 \mathrm{Gyr})$ population. Caputo et al. (1999) and Gallart et al. (1999a) conducted the first comprehensive studies of Leo I's SFH using CMDs obtained with HST/WFPC2. Because these CMDs reached the mainsequence turnoff of the oldest $(>10 \mathrm{Gyr}$ ) populations, they were able to study the multiple stellar populations and complex SFH. Leo I was thought to be unique among the MW satellite dSphs for lacking a conspicuous horizontal branch (HB) until a $12^{\prime} \times 12^{\prime}$ ground-based survey of Leo I by Held et al. (2000) revealed an $\mathrm{HB}$ structure in its CMD. The existence of both an extended blue HB and RR Lyrae stars (Held et al. 2001) suggested that Leo $\mathrm{I}$ is in fact similar to other local dSph galaxies in having a $>10 \mathrm{Gyr}$ population, but the majority of stars were still believed to have formed later than 7 Gyr ago. However, a recent CMD obtained with HST/Advanced Camera for Surveys/Wide Field Camera (Smecker-Hane et al. 2009) reached far deeper than the earlier ones and showed that at least half of the stars were in fact formed more than 9 Gyr ago, which is consistent with the abundant RR Lyrae stars found by Held et al. (2001). In addition, Smecker-Hane et al. combined their CMD with the spectroscopic MDF of Bosler et al. (2007) to find that Leo I experienced two episodes of star formation around 2 and 5 Gyr ago.

Because our chemical evolution models halt when the gas mass drops to zero, we are unable to recover the later phases of SFH (i.e., the two bursts at 2 and 5 Gyr ago). Nonetheless, our model provides insights into the early phase with better time resolution. Overall, our model matches the observed trend of $[\alpha / \mathrm{Fe}]$ with $[\mathrm{Fe} / \mathrm{H}]$ fairly well, but the model MDF slightly overpredicts the frequency of metal-rich stars. The observed MDF also shows a more pronounced peak at $[\mathrm{Fe} / \mathrm{H}]=-1.4$ than the model. The initial starburst that likely led to the formation of Leo I lasted for about $1.4 \mathrm{Gyr}$. This is much shorter than the star formation duration of $\sim 5$ Gyr derived by photometric studies. As with other galaxies in our sample, adding burstiness to our model would help resolve these discrepancies. Lanfranchi \& Matteucci (2010) suggested that Leo I is characterized by a low SFR and intense galactic wind. The main difference between their model and ours is that we start with a much higher gas mass (by a factor of $\sim 400$ ). Also, our model requires a highly efficient SFR to match the observed MDF. The discrepancies with Lanfranchi \& Matteucci partly result from our choice to use unenhanced galactic winds. Metal-enhanced winds would reduce the amount of gas required to be blown out. As for Fornax, our model is qualitatively consistent with previously derived SFHs in the sense that the overall metallicity increases quickly at early times.

Leo I's orbital dynamics, as studied by Sohn et al. (2007) and Mateo et al. (2008), indicate close passes to the center of the MW. The dSph almost certainly lost stars in tidal interactions near its perigalacticon. The prevalence of an intermediate-aged (rather than old) population in Leo I may be a consequence of this tidal stripping. Because the stripped stars do not fall in our spectroscopic sample, our model does not represent some stars that formed early in Leo I's history (see Section 2.8, item 9).

\subsection{Sculptor}

Our chemical evolution model for Sculptor produces one of the best fits to the abundance distributions (Figure 4) out of all of the dSphs, particularly for the asymmetrical MDF. In Paper III, we could not reproduce the width of Sculptor's MDF with an analytical model of chemical evolution. Our more sophisticated model, which more properly treats Fe as a secondary nucleosynthetic product with multiple origins (Types II and Ia SNe), yields a broad, well-matched MDF for the appropriate choice of parameters. The combination of a low SFR normalization $\left(A_{*}\right)$ and low initial gas mass maintains a lower rate of star formation than Fornax or Leo I. Consequently, the metal enrichment is less rapid and the $\mathrm{SN}$-induced gas blowout is less severe. The resulting MDF has both metal-poor and metalrich stars and is less-peaked than for the more luminous dSphs.

Norris \& Bessell (1978) first drew attention to the possibility that Sculptor was chemically inhomogeneous. Da Costa (1984) found that the bulk of Sculptor's stars are slightly younger than the oldest GCs but older than Fornax. With HST/WFPC2 photometry, Monkiewicz et al. (1999) found that Sculptor is just as old as the GCs. Neither study could determine whether the bluer stars were a younger population or blue stragglers from the older population. Mapelli et al. (2009) presented evidence that the blue stars are true blue stragglers, meaning that Sculptor has only an old population. However, old does not necessarily mean single-aged. In fact, Majewski et al. (1999) found that Sculptor undoubtedly contains multiple stellar populations based on its $\mathrm{HB}$ and red giant branch (RGB) morphologies. The existence of a metallicity spread, the depression of $[\alpha / \mathrm{Fe}]$ with increasing metallicity, and the radial change in HB morphology mean that star formation lasted for at least as long as the lifetime of a Type Ia SN and possibly for a few Gyr (Tolstoy et al. 2003; Babusiaux et al. 2005).

Our chemical evolution model conforms to the photometric description of Sculptor's SFH. According to our model, Sculptor formed stars for $1.1 \mathrm{Gyr}$. In fact, one of the major advantages of an abundance-derived SFH is that it can resolve ages of old populations much more finely than a photometricallyderived SFH. As a result, we believe our estimate of the star formation duration to be the most precise presently available for Sculptor.

Lanfranchi \& Matteucci (2004) also found a chemical evolution model to match the five stars with then-available multielement abundance measurements (Shetrone et al. 2003). Their model showed a sharp kink or knee at the time when Type Ia $\mathrm{SNe}$ ejecta began to dilute the $[\alpha / \mathrm{Fe}]$ ratio with large amounts of Fe. Our model shows a less pronounced knee that occurs 

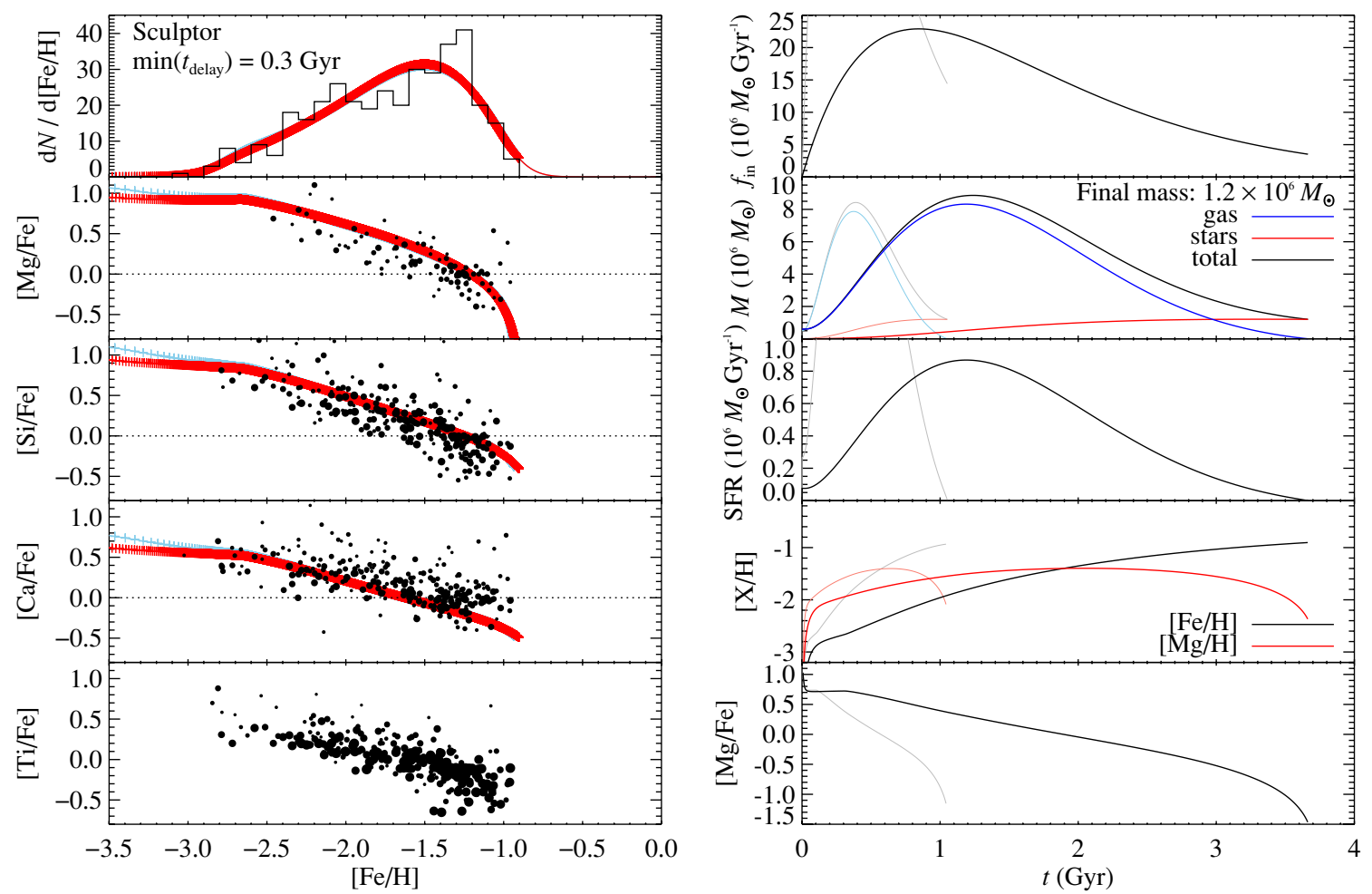

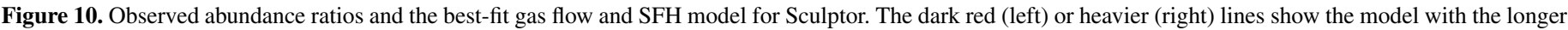
minimum Type Ia SN delay time of $0.3 \mathrm{Gyr}$ (Section 4.1). The light blue (left) or faded (right) lines show the original value of 0.1 Gyr, as in Figure 4.

(A color version of this figure is available in the online journal.)

at lower $[\mathrm{Fe} / \mathrm{H}]$ and higher $[\alpha / \mathrm{Fe}]$ primarily due to our different treatments of the Type Ia SN DTD. Revaz et al. (2009) modeled unpublished abundance measurements by the Dwarf Abundances and Radial Velocities Team (DART) for Sculptor with a sophisticated hydrodynamical model. They found that nearly all of the stars formed between 10 and 14 Gyr ago, with nearly half of the stars forming at least $13 \mathrm{Gyr}$ ago. The model supposed that the stars formed in about five bursts. It is possible that adding burstiness to our model would help to reconcile the model with the observed data, such as the peak in the MDF at $[\mathrm{Fe} / \mathrm{H}]=-1.3$ and the discrepancy in $[\mathrm{Ca} /$ $\mathrm{Fe}]$ at high metallicity. However, Revaz et al.'s model predicted many more stars at $[\mathrm{Fe} / \mathrm{H}]<-3$ than we or DART (who sample a wider area) observe. A less intense initial burst (crudely approximated by the 0.3 Gyr SFR rise time in Figure 4) better matches the low-metallicity MDF. Finally, in constructing a chemical evolution model of Sculptor, Fenner et al. (2006) found that neutron-capture elements contribute significantly to the ability to discriminate between different models of star formation. Large, high-resolution surveys will add these elements to the dSphs' repertoire of abundance measurements.

Like Fornax, the central regions of Sculptor are dominated by a more metal-rich population than the outer regions (Battaglia et al. 2008). Our sample is centrally concentrated in order to maximize the sample size. The selection results in a bias toward metal-rich, presumably younger stars, possibly shortening the derived the SF duration compared to what we would deduce from a more radially extended sample.

We also presented Sculptor's abundance distributions in Paper I (Kirby et al. 2009). Minor modifications to the abundance measurements (Paper II) and the restriction of the plot to points with measurement uncertainties less than $0.3 \mathrm{dex}$ in either axis cause Figure 4 to appear slightly different from
Figures 10-12 in Paper I. The differences do not affect any of the conclusions of Paper I.

\subsection{Leo II}

The abundance distributions for Leo II resemble Sculptor in many ways. The MDF slowly rises to a peak followed by a sharp cutoff, and $[\alpha / \mathrm{Fe}]$ declines smoothly with increasing $[\mathrm{Fe} / \mathrm{H}]$. The best-fit SFH model shows a great deal of gas loss, like Sculptor. Bosler et al. (2007) also suggested that Leo II may have experienced more intense galactic winds than Leo I due to a lower peak in the MDF. In fact, we find that the mass lost per $\mathrm{SN}\left(A_{\text {out }}\right)$ is higher in Leo II $\left(6.6 \times 10^{3} M_{\odot} \mathrm{SN}^{-1}\right)$ than in Leo I $\left(3.9 \times 10^{3} M_{\odot} \mathrm{SN}^{-1}\right)$.

Perhaps by virtue of its large Galactocentric distance $(221 \mathrm{kpc})$, Leo II has maintained star formation for longer than Sculptor. Mighell \& Rich (1996) found from HST/WFPC2 photometry that the dSph started forming stars $14 \mathrm{Gyr}$ ago and continued forming stars for about $7 \mathrm{Gyr}$. In a reanalysis of the same data, Orban et al. (2008) determined that $30 \%$ of Leo II's stars formed earlier than $10 \mathrm{Gyr}$ ago and $67 \%$ formed between 5 and $10 \mathrm{Gyr}$ ago. Shetrone et al. (2009) resolved the age-metallicity degeneracy in the CMD by using metallicities based on spectral synthesis of Keck/LRIS spectra. They found a significant population of stars as young as $3 \mathrm{Gyr}$. However, they pointed out a number of caveats that may introduce large errors into their age measurements.

We derive a star formation duration of $1.6 \mathrm{Gyr}$. Although it is the longest duration that we measure for the eight dSphs, it does not approach the photometrically derived durations. The smoothness of the modeled SFR may mask the true duration of SF. The abundance distributions - particularly $[\mathrm{Si} / \mathrm{Fe}]$ and $[\mathrm{Ti} / \mathrm{Fe}]$ - show a smattering of points beyond the main trend 

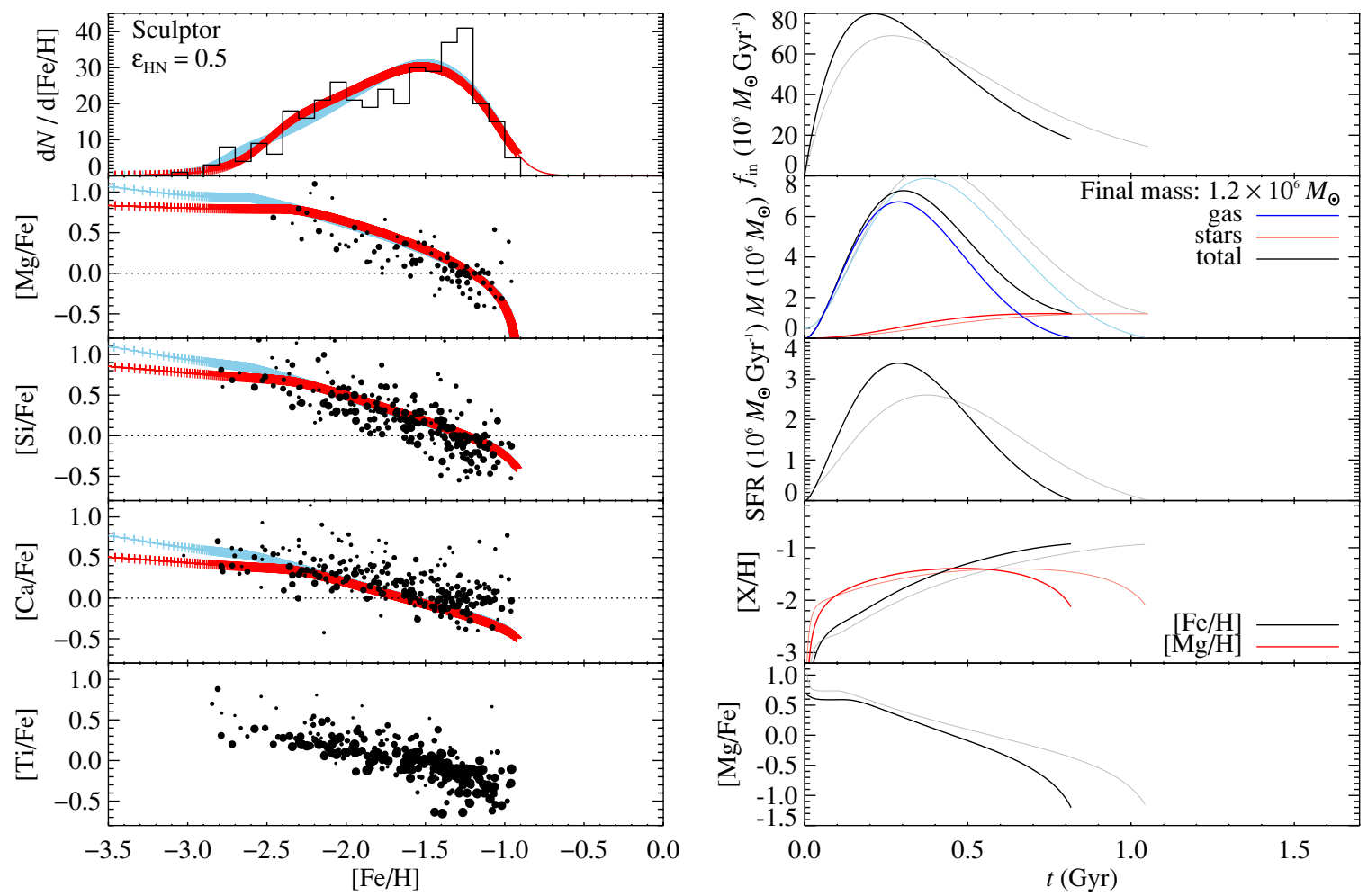

Figure 11. Observed abundance ratios and the best-fit gas flow and SFH model for Sculptor. The dark red (left) or heavier (right) lines show the model with a hypernova fraction of $\epsilon_{\mathrm{HN}}=0.5$ (Section 4.2). The light blue (left) or faded (right) lines show the original value of $\epsilon_{\mathrm{HN}}=0$, as in Figure 4.

(A color version of this figure is available in the online journal.)
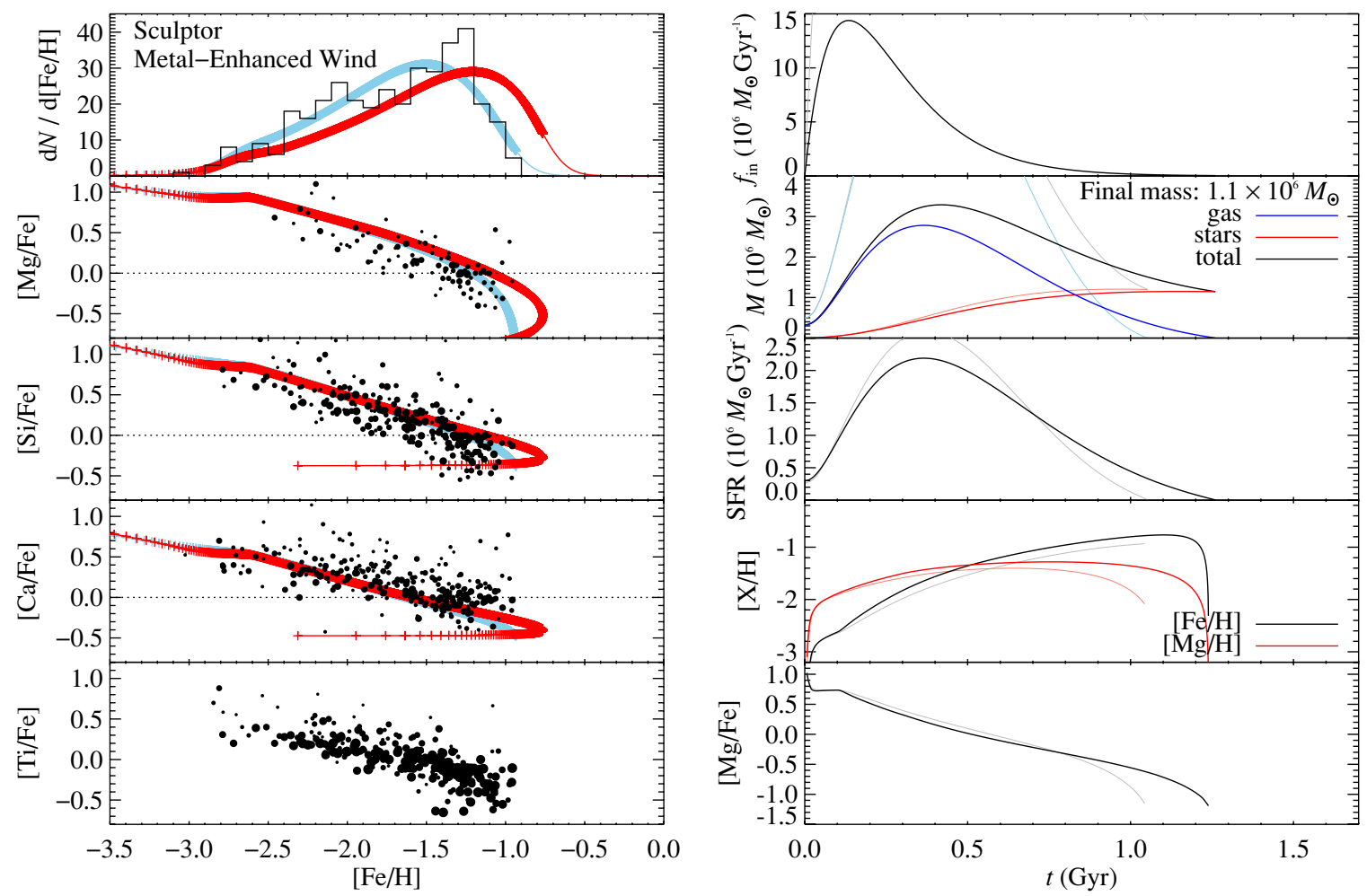

Figure 12. Observed abundance ratios and the best-fit gas flow and SFH model for Sculptor. The dark red (left) or heavier (right) lines show the model with a metal-enhanced wind (Section 4.3). The light blue (left) or faded (right) lines show the original model with an unenhanced wind, as in Figure 4.

(A color version of this figure is available in the online journal.)

line. These stars may represent stellar populations of temporally separated bursts. Revaz et al. (2009) showed that a model with about $13 \mathrm{SF}$ episodes matches the dispersion in $[\mathrm{Mg} / \mathrm{Fe}]$ at a given $[\mathrm{Fe} / \mathrm{H}]$ (observations by Shetrone et al. 2009) fairly well. 
Our model for Leo II, like Sculptor, may benefit by adding burstiness.

\subsection{Sextans}

Sextans, Draco, and Ursa Minor form a class of galaxies with similar abundance distributions and SFH models. Their MDFs are fairly symmetric (less so for Ursa Minor) with a clump of stars at $[\mathrm{Fe} / \mathrm{H}] \sim-3$. Their $[\alpha / \mathrm{Fe}]$ ratios decline smoothly with increasing $[\mathrm{Fe} / \mathrm{H}]$. The dispersion in $[\alpha / \mathrm{Fe}]$ at a given $[\mathrm{Fe} / \mathrm{H}]$ is fairly small. Most of the derived star formation parameters are similar (infall normalization, $A_{\text {in }} \sim(1.1-1.5) \times 10^{9} \mathrm{Gyr}$; infall timescale, $\tau_{\text {in }} \sim 0.2$; outflow rate, $A_{\text {out }} \sim 10^{4} M_{\odot} \mathrm{SN}^{-1}$ ).

The small bump in the MDF at $[\mathrm{Fe} / \mathrm{H}] \sim-3$ deserves some discussion because it appears in Sextans, Draco, and Ursa Minor. A depression in the MDF appears between the bump and the bulk of the MDF. This bump might indicate a small, rapid SF burst at early times followed by an epoch of minimal star formation, possibly because the $\mathrm{SNe}$ from the initial burst blew out the gas. When the galaxy reacquired more cool gas, the bulk of SF began. The few available $[\alpha / \mathrm{Fe}]$ measurements in the bump are large, indicating that the stars in the bump formed before the onset of Type Ia SNe. Because our model does not permit individual bursts, we cannot support this speculation beyond our qualitative argument.

Despite the low metallicity and low luminosity of Sextans, Bellazzini et al. (2001) found that the dSph has at least two stellar populations based on its HB and RGB morphology. With HST/WFPC2 photometry, Orban et al. (2008) found no stars older than 10 Gyr. Lee et al. (2009) measured Sextans's SFH based on wide field photometry coupled with an algorithm that self-consistently derives the SFH and chemical evolution of the galaxy. They deduced that SF in Sextans occurred mainly between 11 and 15 Gyr ago, but some stars formed as recently as $8 \mathrm{Gyr}$ ago. However, they assumed that Sextans is a closed box. In Paper III, we showed that the MDF is inconsistent with a closed box. We allow gas to leave the system, which would bring an earlier end to SF than in a closed box. As a result, we find an SF duration of just $0.8 \mathrm{Gyr}$.

\subsection{Draco}

Because we conducted a more intense observational campaign on Draco than on Sextans, we sample better Draco's abundance space. The better sampling does not change our qualitative description of the trio comprised of Sextans, Draco, and Ursa Minor (see Section 3.5). The metal-rich side of Draco's MDF seems tiered, with fewer stars than our model predicts at $[\mathrm{Fe} / \mathrm{H}]=-1.5$ and -1.2 . The tiers may indicate discontinuous periods of SF.

As a consequence of its proximity, Draco was one of the first dSphs subjected to spectroscopic scrutiny. This system has a stellar mass comparable to GCs, which are homogeneous in iron-peak elements. Therefore, the discovery of a metal abundance spread within this system (Kinman \& Kraft 1980; Kinman et al. 1981; Stetson 1984; Smith 1984; Lehnert et al. 1992) proved to be a notable peculiarity. Furthermore, Draco contains stars more metal-poor than any GC. The first attempt to interpret the metallicity distribution within Draco was that of Zinn (1978). He compared metallicities derived for 23 red giants from the Hale $5 \mathrm{~m}$ multichannel scanner to a chemical evolution model that incorporated gas loss (with a rate proportional to the SFR) but no gas inflow. In order to account for the low metallicity of Draco, Zinn (1978) inferred that this system had lost some
90\%-99\% of its initial gas mass. Subsequent spectroscopic and photometric work has more extensively documented the MDF and increased the number of elements for which abundances have been measured (Shetrone et al. 1998, 2001a; Aparicio et al. 2001; Bellazzini et al. 2002; Winnick 2003; Smith et al. 2006; Faria et al. 2007; Abia 2008; Cohen \& Huang 2009).

HST/WFPC2 photometry (Grillmair et al. 1998) and widefield Isaac Newton Telescope photometry (Aparicio et al. 2001) showed little evidence for stars younger than 10 Gyr in Draco. On the other hand, Ikuta \& Arimoto (2002), who also pointed out the similarities between Sextans, Draco, and Ursa Minor, found a longer SF duration: between 3.9 and 6.5 Gyr. However, Ikuta \& Arimoto, like Lee et al. (2009), assumed that a closed box was an adequate description of the galaxy. In Paper III, we determined that failing to account for gas outflow overpredicts the peak metallicity of the MDF and that failing to account for gas infall results in an MDF shape that does not match the observations. Our abundance-based SF duration, relaxing the closed box assumption, is 0.7 Gyr. Strangely, based on the same HST/WFPC2 data that Grillmair et al. used, Orban et al. (2008) determined that half of the stars in Draco are younger than 10 Gyr. Orban et al. derived SFHs for many dSphs, and they did not mention Draco explicitly in their text. As a result, we do not know why their SFH diverged from that of Grillmair et al.

Cohen \& Huang (2009) analyzed high-resolution spectroscopic abundances for eight newly observed stars and six stars from the literature. They fit a toy model with low- and highmetallicity plateaus in $[\mathrm{X} / \mathrm{Fe}]$. The low-metallicity plateau has a maximum metallicity of $[\mathrm{Fe} / \mathrm{H}]=-2.9$ for $[\mathrm{Mg} / \mathrm{Fe}]$ and -2.4 for $[\mathrm{Si} / \mathrm{Fe}]$. We do not see a low-metallicity plateau because our sample does not include enough metal-poor stars. Instead, we observe a smooth, monotonic decline in all four $[\alpha / \mathrm{Fe}]$ ratios as a function of increasing $[\mathrm{Fe} / \mathrm{H}]$. The absence of a low-metallicity plateau for the metallicity range of our sample suggests that Type Ia SNe were exploding for nearly the entire SF lifetime of Draco.

Marcolini et al. (2006, 2008) constructed a hydrodynamical model of a Draco-like $\mathrm{dSph}$. In order for $[\alpha / \mathrm{Fe}]$ to drop to 0.2 dex, their modeled dSph must have evolved for at least 2 Gyr. However, at small radius - the location of most spectroscopic surveys, including the majority of our Draco sample- $[\alpha / \mathrm{Fe}]$ does drop to lower values sooner than in the dSph as a whole. Nonetheless, Marcolini et al. predicted mostly stars with $[\alpha / \mathrm{Fe}]$ larger than 0.2 dex with a plateau at low metallicity. We observe neither of these qualities. Nonetheless, their model does qualitatively reproduce important features of $\mathrm{dSph}$ abundance distributions, including radial gradients in both $[\mathrm{Fe} / \mathrm{H}]$ and $[\alpha / \mathrm{Fe}]$, the shape of the MDF, and an anti-correlation between metallicity and velocity dispersion.

Finally, we point out that, according to our model, Draco lost an enormous amount of gas from $\mathrm{SN}$ winds during its $\mathrm{SF}$ lifetime. Lanfranchi \& Matteucci (2007) used Draco and Ursa Minor as case studies in the importance of SN winds. One interesting divergence from our model is that they found that a wind intensity proportional to the SFR rather than the SN rate better voided the $\mathrm{dSph}$ of gas by the present time, in agreement with the observed absence of gas. Our different prescription for the Type Ia DTD may mitigate the difference between the SFR and $\mathrm{SN}$ rate.

\subsection{Canes Venatici I}

Of all of our dSph models, that for Canes Venatici I adheres most closely to the observed abundance distributions, in part 
because of the sparse sampling. The MDF is a perfect match, and the predicted $[\alpha / \mathrm{Fe}]$ line passes through the observed locus of points, except for veering to slightly high $[\alpha / \mathrm{Fe}]$ values at high $[\mathrm{Fe} / \mathrm{H}]$. Unfortunately, only three stars pass the $[\mathrm{Mg} / \mathrm{Fe}]$ uncertainty cut of $0.3 \mathrm{dex}$. More measurements of [Si/Fe] and $[\mathrm{Ca} / \mathrm{Fe}]$ help us to determine an SF duration of $0.9 \mathrm{Gyr}$ and an unusually low SFR exponent of $\alpha=0.36$. The weaker dependence on gas mass shapes the SFR profile in such a way that produces a more symmetric MDF while preserving a steadily declining $[\alpha / \mathrm{Fe}]$ distribution with increasing $[\mathrm{Fe} / \mathrm{H}]$.

Because Canes Venatici I was discovered recently (Zucker et al. 2006), few photometric studies exist. Martin et al. (2008b) found that the dSph contains mostly stars older than $10 \mathrm{Gyr}$, but $5 \%$ of the stars could be as young as 1.4 Gyr. Kuehn et al. (2008), with a shallower CMD, found possible evidence for a population as young as $0.6 \mathrm{Gyr}$. They also found three candidate anomalous Cepheid variables, indicating an intermediate-age population. Because the young population is much smaller than the old population, our chemical evolution model and its SF duration should be viewed as applicable to the dominant old population.

\subsection{Ursa Minor}

The low-mass Ursa Minor dSph has sometimes been studied in comparison with the Draco dSph, in regard to both its metallicity inhomogeneity and stellar population (Zinn 1981; Stetson 1984; Bell 1985; Shetrone et al. 2001b; Bellazzini et al. 2002; Winnick 2003; Abia 2008). A relatively small age spread and an ancient mean age (Olszewski \& Aaronson 1985; Mighell \& Burke 1999; Carrera et al. 2002) also makes it an interesting contrast to halo GCs. However, spectroscopy has shown that Ursa Minor has a heavy element abundance spread of more than 1 dex (Zinn 1981; Shetrone et al. 2001a; Winnick 2003; Sadakane et al. 2004; Cohen \& Huang 2010) even though its stellar mass is similar to that of a GC.

Cudworth et al. (1986) conducted a photometric survey of Ursa Minor down to the HB. With $\sim 450$ members, they found that the stellar population resembles that of an old, metal-poor GC with a steep RGB and a blue horizontal branch. The HST/ WFPC2 imaging study of Mighell \& Burke (1999) confirmed this SFH: a single major burst of star formation about $14 \mathrm{Gyr}$ ago with a duration of less than 2 Gyr. Our best-fit model agrees with these earlier results. From our observed abundance distributions, we deduce that almost all of the star formation in Ursa Minor occurred over an interval of only 0.4 Gyr. In contrast, Ikuta \& Arimoto (2002) derived an extended period of star formation lasting for about 5 Gyr from their closed-box analysis of the CMD. In Paper III, we showed that Ursa Minor's MDF is inconsistent with a closed box. Cohen \& Huang (2010) used metallicities from moderate resolution spectra combined with ages from isochrones to reaffirm that most of the stars in Ursa Minor are quite old.

MDFs have been generated from photometric surveys by Bellazzini et al. (2002) and from moderate resolution spectroscopy by Winnick (2003). That of Bellazzini et al. (2002) is a good match to our observed MDF given in Figure 9. Both show a sharp rise to a peak metallicity of about -2 dex with a more gradual decline towards higher $[\mathrm{Fe} / \mathrm{H}]$. The best fit chemical evolution model for Ursa Minor produces an MDF that fails to match the rapid rise seen at $[\mathrm{Fe} / \mathrm{H}] \lesssim-2.3$ dex.

Cohen \& Huang (2010) provided detailed abundance analyses for a sample of 16 RGB stars, 6 of which came from earlier work by Shetrone et al. (2001a) or from Sadakane et al. (2004). Their trends for $[\mathrm{Mg} / \mathrm{Fe}],[\mathrm{Si} / \mathrm{Fe}],[\mathrm{Ca} / \mathrm{Fe}]$, and $[\mathrm{Ti} / \mathrm{Fe}]$ agree qualitatively with those found here, but their sample has better coverage of the regime $[\mathrm{Fe} / \mathrm{H}]<-2.5 \mathrm{dex}$, where they found a plateau in $[\alpha / \mathrm{Fe}]$. At very low metallicity, $[\alpha / \mathrm{Fe}]$ in our models reaches highly supersolar ratios, which are larger than those observed at the metal-poor end of the Ursa Minor population by Cohen \& Huang.

Previous chemical evolution models of Ursa Minor include those of Lanfranchi \& Matteucci (2004), who found that Ursa Minor has the shortest duration of star formation of any of the six dSph satellites they studied. They deduced that Ursa Minor experienced only a single burst lasting perhaps $3 \mathrm{Gyr}$, a moderately high star formation efficiency, and an intermediate wind efficiency. In our model the wind efficiency, $A_{\text {out }}$, is the highest of all the dSphs in our sample (see Table 2). Lanfranchi \& Matteucci's predicted MDF fails at low $[\mathrm{Fe} / \mathrm{H}]$, as does ours, by being too extended. In a later paper, Lanfranchi \& Matteucci (2007) studied the effect of galactic winds. They concluded that a strong galactic wind is necessary to reproduce the rather low $[\mathrm{Fe} / \mathrm{H}]$ of the peak of the Ursa Minor MDF, but they still failed to reproduce the sudden scarcity of stars more metal-poor than the MDF peak.

Both Martínez-Delgado et al. (2001) and Muñoz et al. (2005) have discovered tidal debris around Ursa Minor. As we discussed in Section 2.8 (item 9), our observations are centrally concentrated and therefore biased toward the relatively younger, more metal-rich population that is still bound to the $\mathrm{dSph}$. A truly complete analysis of Ursa Minor's SFH must also include the tidally stripped, unbound stars.

\section{FURTHER EXPLORATION OF THE CHEMICAL EVOLUTION MODEL}

In this section, we explore the parameters of the chemical evolution model that were previously not allowed to vary. Namely, we examine the dependence of the outcome of the model on the Type Ia SN DTD, the hypernova fraction, and the metal enhancement of SN winds. We have chosen Sculptor as a case study. In each of the following three sections, we alter one aspect of the chemical evolution model for Sculptor. Then, we use Powell's method to find the combination of the six free parameters that maximizes the likelihood estimator, as before. A Monte Carlo Markov Chain of at least $10^{4}$ trials provides the two-sided $68.3 \%$ confidence intervals for the first two altered models. Table 4 compares the results of the new models with the original model.

\subsection{Type Ia Delay Time Distribution}

We have adopted the Type Ia DTD of Maoz et al. (2010). The model is very sensitive to the delay time of the first Type Ia SN to explode after the onset of star formation. Unfortunately, this quantity is poorly measured. We have chosen 0.1 Gyr because that is the maximum value that Maoz et al.'s DTD seems to allow. However, the DTD was measured in a range of galaxies with widely varying star formation environments. The details of the combined DTD (Figure 1) may not be appropriate for dSphs. For example, Kobayashi et al. (1998) and Kobayashi \& Nomoto (2009) suggested that single-degenerate Type Ia SNe will be inhibited at low metallicity $([\mathrm{Fe} / \mathrm{H}] \lesssim-1)$. Nonetheless, the decline of $[\alpha / \mathrm{Fe}]$ with increasing $[\mathrm{Fe} / \mathrm{H}]$ in Figures 3-9 demand that some kind of Type Ia SN explode. Thus, the lowmetallicity Type Ia SNe in dSphs may be mergers of doubledegenerate binaries only. The removal of the single-degenerate channel could affect the DTD. 
Table 4

Sensitivity of Sculptor Model Parameters to Assumptions

\begin{tabular}{lcccc}
\hline \hline Parameter & Baseline & $\min \left(t_{\text {delay }}\right)=0.1 \mathrm{Gyr}$ & $\epsilon_{\mathrm{HN}}=0.5$ & Metal-enhanced Wind \\
\hline$A_{*}\left(10^{6} M_{\odot} \mathrm{Gyr}^{-1}\right)$ & $0.47_{-0.12}^{+0.09}$ & $0.12_{-0.06}^{+0.03}$ & $0.68_{-0.18}^{+0.10}$ & 0.85 \\
$\alpha$ & $0.83_{-0.08}^{+0.14}$ & $0.93_{-0.09}^{+0.30}$ & $0.84_{-0.06}^{+0.16}$ & 0.93 \\
$A_{\text {in }}\left(10^{9} M_{\odot} \mathrm{Gyr}^{-1}\right)$ & $0.70_{-0.08}^{+0.12}$ & $0.07_{-0.01}^{+0.01}$ & $1.02_{-0.14}^{+0.15}$ & 0.29 \\
$\tau_{\text {in }}(\mathrm{Gyr})$ & $0.27_{-0.02}^{+0.02}$ & $0.84_{-0.06}^{+0.06}$ & $0.21_{-0.01}^{+0.02}$ & 0.13 \\
$A_{\text {out }}\left(10^{3} M_{\odot} S N^{-1}\right)$ & $5.36_{-0.17}^{+0.16}$ & $5.38_{-0.21}^{+0.18}$ & $5.14_{-0.17}^{+0.13}$ & 0.53 \\
$M_{\text {gas }}(0)\left(10^{6} M_{\odot}\right)$ & $0.50_{-0.25}^{+0.62}$ & $0.60_{-0.32}^{+1.01}$ & $0.00_{-0.00}^{+0.17}$ & 0.33 \\
SF duration $(\mathrm{Gyr})$ & 1.05 & 3.66 & 0.82 & 1.26
\end{tabular}

Notes. We were unable to compute uncertainties for the Metal-enhanced Wind model because the model is numerically unstable to small perturbations. The SF duration is a derived value, not a free parameter, and we did not calculate its uncertainty.

In order to explore the impact of changing the DTD on the chemical evolution model, we have recomputed the most-likely model parameters for Sculptor with a minimum Type Ia delay time of $0.3 \mathrm{Gyr}$ instead of $0.1 \mathrm{Gyr}$. We did not change the DTD normalization. Figure 10 shows the result compared to the original model (Figure 4). The abundance distribution is identical except for the low-metallicity $[\alpha / \mathrm{Fe}]$ plateau, which is flatter for the longer delay time because the mass dependence of the Type II SN yields is muted. However, the right panel of Figure 10 shows that the SFH has changed dramatically. In particular, the timescale of SF has been expanded. In fact, the differences in the SFHs can be explained by multiplying the time variable in the original model by about 3.5. The result is less intense star formation over a longer time. In the end, just as many stars are formed and just as much gas is blown out as in the original model.

We conclude that the Type Ia SN DTD is a major uncertainty in our model. The abundance data alone does not help to determine the minimum delay time. The timescales in our models can be multiplied by a factor constrained only by the poorly known minimum Type Ia SN delay time.

\subsection{Hypernova Fraction}

SN 1998bw was immediately identified to be unusual because of its association with a gamma ray burst and a light curve that suggested relativistically expanding gas (Galama et al. 1998). Iwamoto et al. (1998) determined that the explosion energy for SN 1998bw was about 30 times larger than the average SN. The energy of the explosion has consequences for the nucleosynthesis. Nomoto et al. (2006) calculated nucleosynthetic yields for $\mathrm{SNe}$ at a variety of explosion energies.

One of the fixed parameters in our model is the fraction of stars that explode as very energetic $\mathrm{HNe}\left(\epsilon_{\mathrm{HN}}\right)$. We initially chose $\epsilon_{\mathrm{HN}}=0$ (no HNe) because it seemed to better match the abundance patterns at the lowest metallicities (e.g., $[\mathrm{Ca} / \mathrm{Fe}]$ in Sculptor). In order to explore the effect of HNe, we have also found the most likely model for Sculptor with $\epsilon_{\mathrm{HN}}=0.5$. This is the value that Nomoto et al. chose for their own chemical evolution model of the solar neighborhood. Romano et al. (2010) further explored the effect of changing $\epsilon_{\mathrm{HN}}$.

Figure 11 compares the result of the model with $\epsilon_{\mathrm{HN}}=0.5$ with the original model $\left(\epsilon_{\mathrm{HN}}=0\right)$. The abundance distributions are nearly identical except at $[\mathrm{Fe} / \mathrm{H}]<-2.3$. The model with larger $\epsilon_{\mathrm{HN}}$ reaches higher $[\mathrm{Fe} / \mathrm{H}]$ before Type Ia $\mathrm{SNe}$ turn-on. This ensures that the lowest metallicity stars are not polluted by Type Ia SNe ejecta. The result is a plateau in $[\alpha / \mathrm{Fe}]$ at low $[\mathrm{Fe} / \mathrm{H}]$. We further discuss the presence of such a plateau in the
$[\mathrm{Ca} / \mathrm{Fe}]$ ratio of Sculptor and the absence of plateaus in other dSphs in Section 5.1.1.

The effect on the SFH is more noticeable than on the abundance distributions. The total star formation duration shortens to $0.82 \mathrm{Gyr}$ from $1.1 \mathrm{Gyr}$. The $\mathrm{HN}$ model also requires no initial gas, though the original model for Sculptor already did not require very much gas. Less gas is lost to $\mathrm{SN}$ winds in the $\mathrm{HN}$ model.

In conclusion, the inclusion of $\mathrm{HNe}$ has a minor effect on the abundance distributions and $\mathrm{SFH}$. The most notable result is that very metal-poor stars $([\mathrm{Fe} / \mathrm{H}]<-2.3)$ in the $\mathrm{HN}$ model have $[\alpha / \mathrm{Fe}]$ ratios that are inconsistent with any amount Type Ia SN ejecta. Instead, these stars incorporate the ejecta of only Type II SNe or HNe.

\subsection{Metal-enhanced Supernova Winds}

The $\mathrm{SNe}$ in our model expel gas without regard to its composition. However, SN winds might be expected to be more metal-rich than the average gas-phase metallicity because metals are more opaque (and therefore more susceptible to radiation pressure) than hydrogen and helium and because the same $\mathrm{SNe}$ that create the metals could blow them away (Vader 1986; Mac Low \& Ferrara 1999). In this section, we explore the effect of a metal-enhanced SN wind. We refer the reader to Robertson et al. (2005) for a more thorough discussion of a model that included metal-enhanced winds from dwarf galaxies.

We paramaterize the metallicity dependence of the wind by $f_{Z}$, which can vary between 0 and 1 . Thus, we replace Equation (15) with

$\dot{\xi}_{j, \text { out }}= \begin{cases}A_{\text {out }} X_{j}\left(\dot{N}_{\text {II }}+\dot{N}_{\text {Ia }}\right)\left(1-f_{Z}\right) & j=\mathrm{H}, \mathrm{He} \\ A_{\text {out }} X_{j}\left(\dot{N}_{\text {II }}+\dot{N}_{\text {Ia }}\right)\left[f_{Z}\left(\frac{1}{Z}-1\right)+1\right] & \text { otherwise }\end{cases}$

If $f_{Z}=0$, then the wind is unenhanced. If $f_{Z}=1$, then the winds expel only metals and no hydrogen or helium. For this experiment, we fix $f_{Z}$ at 0.01 . Although that value seems small, the effect on the SFH is dramatic.

The modeled metallicity distribution (Figure 12) does not fit the observed distribution as well as for the original model. Instead, there is an overabundance of metal-rich stars. The metal-rich discrepancy could be mitigated by increasing $A_{\text {out }}$ (the total amount of gas lost per SN) at the cost of worsening the match at intermediate metallicities. The predicted $[\alpha / \mathrm{Fe}]$ distributions change only at $[\mathrm{Fe} / \mathrm{H}] \gtrsim-1.2$. Metal-enhanced gas loss causes the hook back toward lower $[\mathrm{Fe} / \mathrm{H}]$ in the $[\alpha / \mathrm{Fe}]$ diagrams. Because the SFR is very low by the time 
$[\mathrm{Fe} / \mathrm{H}]$ begins to decrease, very few stars are formed during this time.

The most dramatic effect on the SFH is that much less gas is lost over the lifetime of SF in the metal-enhanced wind model than in the original model. With an unenhanced wind, Sculptor ejects $1.8 \times 10^{8} M_{\odot}$ of the gas that it starts with or accretes. With a metal-enhanced wind, that number decreases to $4.5 \times 10^{6} \mathrm{M}_{\odot}$. In both models, Sculptor forms about $1.2 \times 10^{6} M_{\odot}$ of stars. The implications for galaxy evolution are dramatic. In the first case, over $10^{8} M_{\odot}$ of gas is required to catalyze star formation in Sculptor. Nearly all of this gas is returned to the ISM. In the metal-enhanced wind case, star formation in Sculptor requires a gas mass of only a few times its final stellar mass. The mass of metals returned to the intergalactic medium in both cases is the same, but in the metal-enhanced wind model, the metals in the ejected gas are much more concentrated. Changes to other aspects of the SFH are subtle.

We conclude that the amount of metal enhancement in the SN blowout dramatically affects the gas dynamics of the dSph. Even a $1 \%$ metal enhancement reduces the total amount of gas required for star formation by a factor of 40 . However, a model with $f_{Z}=0.01$ results in a worse match to the observed metallicity distribution than the original model with an unenhanced wind. A lower, non-zero value of $f_{Z}$ might produce better agreement with the observed abundance data while reducing the amount of gas infall required from the unenhanced wind scenario. The literature on galactic chemical evolution contains a diversity of $\mathrm{SN}$ feedback treatments. We refer the reader to the articles we have already mentioned (e.g., Recchi et al. 2001; Lanfranchi \& Matteucci 2004; Robertson et al. 2005; Romano et al. 2006; Marcolini et al. 2008) for more thorough treatments.

\section{TRENDS WITH GALAXY PROPERTIES}

We now discuss trends of the abundance distributions and derived SFH parameters with observed galaxy properties, such as luminosity, velocity dispersion, half-light radius, and Galactocentric distance. We show that luminosity is the only galaxy property that shows any convincing correlation with the properties of the abundance distributions.

\subsection{General $[\alpha / F e]$ Trends}

Figure 13 shows the trend lines of the different element ratios with $[\mathrm{Fe} / \mathrm{H}]$. The trend line is defined by the average of the element ratio, weighted by the inverse square of the measurement uncertainties, in a moving window of 0.5 dex in $[\mathrm{Fe} / \mathrm{H}]$. The moving averages relax the uncertainty cut of 0.3 dex (used for Figures 2-9) to 1 dex, meaning that all of the measurements from the catalog (Paper II) are included. The bottom panel shows the average of four element ratios, which is called $\langle[\alpha / \mathrm{Fe}]\rangle$. The weight of the line fades as fewer stars contribute to the average near the ends of the MDF. The figure legend lists the dSphs in order of decreasing luminosity. For comparison, some panels of the figure also display the trends for the MW halo and disk for available element ratios (Venn et al. 2004). ${ }^{6}$

\footnotetext{
6 The data from Venn et al. (2004) are a compilation of data from the following sources: Bensby et al. (2003), Burris et al. (2000), Edvardsson et al. (1993), Fulbright (2000, 2002), Gratton \& Sneden (1988, 1991, 1994), Hanson et al. (1998), Ivans et al. (2003), Johnson (2002), McWilliam et al. (1995), McWilliam (1998), Nissen \& Schuster (1997), Prochaska et al. (2000), Reddy et al. (2003), Ryan et al. (1996), and Stephens \& Boesgaard (2002).
}

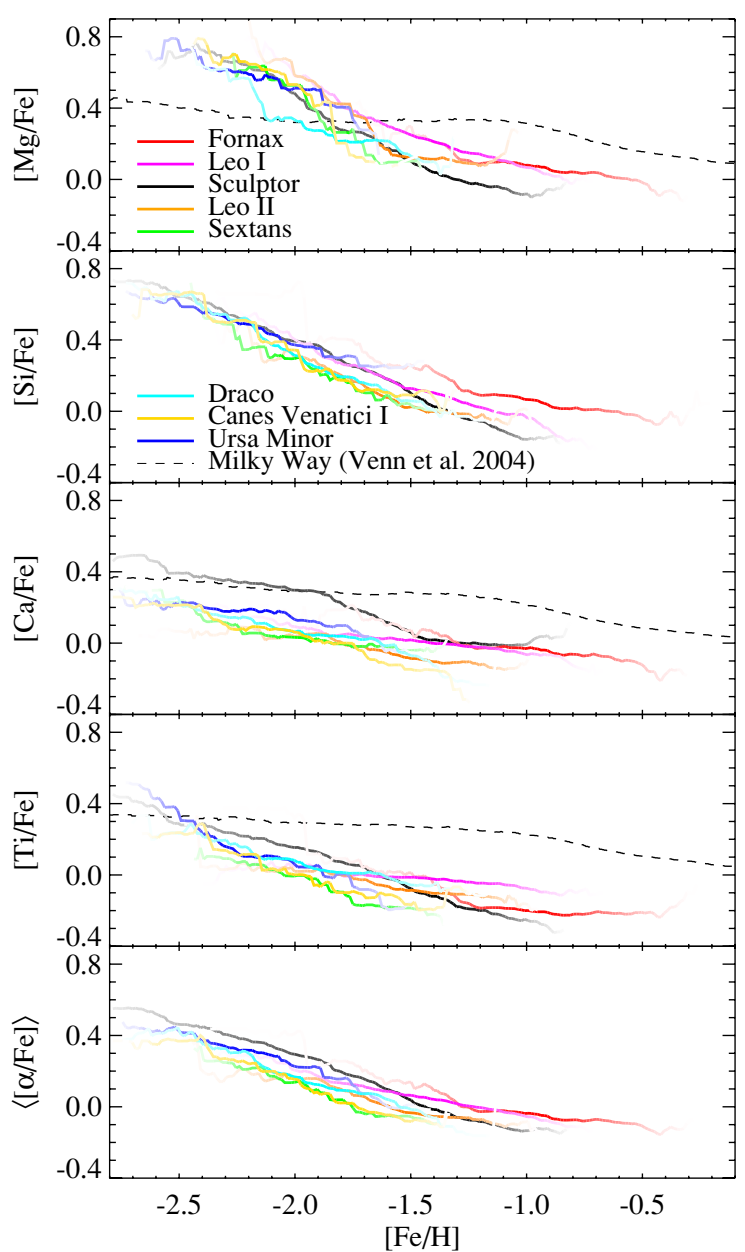

Figure 13. Moving averages, inversely weighted by measurement uncertainty, of abundance ratios for the eight dSphs and for the MW (Venn et al. 2004, who compiled data from the references given in footnote 6). The bottom panel shows $\langle[\alpha / \mathrm{Fe}]\rangle$, the average of the top four panels. The line weight is proportional to the number of stars contributing to the average. The legend lists the dSphs in decreasing order of luminosity. Except for $[\mathrm{Ca} / \mathrm{Fe}]$ in Sculptor, the abundance ratios do not show a low-metallicity plateau, which indicates that Type Ia SNe explode for nearly the entire duration of star formation. Our data are sparse at $[\mathrm{Fe} / \mathrm{H}]<-2.5$, and Type Ia SNe need not explode at times corresponding to those low metallicities. Only the galaxies luminous enough to reach $[\mathrm{Fe} / \mathrm{H}] \gtrsim-1$ eventually achieve an equilibrium between Types II and Ia $\mathrm{SNe}$ and therefore a plateau at high metallicity.

Figure 13 presents the broad trends of the evolution of $[\alpha / \mathrm{Fe}]$ with increasing $[\mathrm{Fe} / \mathrm{H}]$. It does not convey the width of the dispersion of the $[\alpha / \mathrm{Fe}]$ distributions at a given metallicity, nor does it show the details at the margins of the MDF. The extremely metal-poor stars, which represent some of the oldest known stars, are not shown in Figure 13.

\subsubsection{Universal Abundance Pattern in dSphs}

The figure does show that the abundance distributions of dSphs evolve remarkably similarly. Although the dSphs span different ranges of $[\mathrm{Fe} / \mathrm{H}],\langle[\alpha / \mathrm{Fe}]\rangle$ follows roughly the same trend line. This similarity contradicts the reasonable expectation that different $\mathrm{dSphs}$ should show a knee in $[\alpha / \mathrm{Fe}]$ at different values of $[\mathrm{Fe} / \mathrm{H}]$ (e.g., Matteucci \& Brocato 1990; Gilmore \& Wyse 1991; Tolstoy et al. 2009). In fact, Tolstoy et al. did indeed find a knee in at $[\mathrm{Fe} / \mathrm{H}]=-1.8$ in DART's preliminary measurements for $[\mathrm{Ca} / \mathrm{Fe}]$ in Sculptor. Our measurements of $[\mathrm{Ca} / \mathrm{Fe}]$ in Sculptor also show a knee at the same metallicity and the same $[\mathrm{Ca} / \mathrm{Fe}]$. Ursa Minor possibly has a knee in 
$[\mathrm{Ca} / \mathrm{Fe}]$, but with a lower $[\mathrm{Ca} / \mathrm{Fe}]$ plateau. In agreement with Tolstoy et al.'s and others' predictions for lower mass systems to experience less intense SF, Ursa Minor's possible knee occurs at lower $[\mathrm{Fe} / \mathrm{H}]$ than Sculptor's knee. However, the knee is apparent only in $[\mathrm{Ca} / \mathrm{Fe}]$ and only in Sculptor and possibly Ursa Minor. The element ratios that would better identify the onset of Type Ia SNe, $[\mathrm{Mg} / \mathrm{Fe}]$ and $[\mathrm{Si} / \mathrm{Fe}]$, do not show a knee for any dSph.

The lack of knees for $[\mathrm{Fe} / \mathrm{H}]>-2.5$ and the lack of lowmetallicity plateaus in the $[\alpha / \mathrm{Fe}]$ distributions imply that Type Ia SNe exploded throughout almost all of the SFHs of all dSphs. Of course, the very first stars, which have yet to be found, must be free of all SN ejecta. The stars to form immediately after the first SNe must incorporate only Type II SN ejecta. The very lowest metallicity stars in dSphs likely represent this population. Stars with $[\mathrm{Fe} / \mathrm{H}] \gtrsim-2.5$ formed after the Type Ia SNinduced depression of $[\alpha / \mathrm{Fe}]$. We have already explored the possibility of low-metallicity plateaus in $[\mathrm{Ca} / \mathrm{Fe}]$, but we discount the absence of Type Ia SN products as the cause because $[\mathrm{Ca} / \mathrm{Fe}]$ is the only element ratio to show the plateau. We speculate instead that metallicity-dependent Type Ia nucleosynthesis (e.g., Timmes et al. 2003; Howell et al. 2009) might shape the $[\mathrm{Ca} / \mathrm{Fe}]$ distribution differently from the other element ratios.

High-metallicity plateaus can form when the SF achieves a constant rate for a duration long enough for the ratio between Types II and Ia SNe to be constant. The SFR would achieve an equilibrium between the production of $\alpha$ elements and Fe. The value of $[\alpha / \mathrm{Fe}]$ at the plateau depends on the IMF and SN DTD. The SFR need not be strictly constant. As Revaz et al. (2009) pointed out, a bursty SF profile with a high duty cycle can mimic a constant SFR. In that case, we would expect a scatter about the mean value of $[\alpha / \mathrm{Fe}]$ at a given $[\mathrm{Fe} / \mathrm{H}]$, but the mean value would not necessarily evolve with increasing $[\mathrm{Fe} / \mathrm{H}]$. We do observe high-metallicity plateaus, seen in Figure 13. The trends for $[\mathrm{Mg} / \mathrm{Fe}]$ and $[\mathrm{Si} / \mathrm{Fe}]$ do not completely flatten, but the slopes at $[\mathrm{Fe} / \mathrm{H}]>-1$ are less than the slopes at $[\mathrm{Fe} / \mathrm{H}]<-1.5$. The trends for $[\mathrm{Ca} / \mathrm{Fe}]$ and $[\mathrm{Ti} / \mathrm{Fe}]$ do completely flatten for some dSphs. Only the more luminous dSphs, which reached metallicities of $[\mathrm{Fe} / \mathrm{H}] \gtrsim-1.2$, achieved the high-metallicity plateau. The $[\alpha / \mathrm{Fe}]$ ratios of Sextans, Draco, Canes Venatici I, and Ursa Minor do not flatten. We conclude that dSphs with high enough SFRs to reach stellar masses of at least $10^{6} M_{\odot}$ experienced roughly constant $\mathrm{SF}$ at late times, corresponding to metallicities $[\mathrm{Fe} / \mathrm{H}] \gtrsim-1.2$.

Beneath the apparently universal path in $[\alpha / \mathrm{Fe}]-[\mathrm{Fe} / \mathrm{H}]$ space, the abundance trends vaguely group by luminosity. Higher luminosity dSphs tend to have slightly higher values of $[\alpha / \mathrm{Fe}]$ at a given $[\mathrm{Fe} / \mathrm{H}]$ than lower luminosity $\mathrm{dSphs}$. The tracks for Sextans, Draco, and Canes Venatici I tend to lie below the other dSphs. Fornax and Leo I tend to lie above Sculptor and Leo II. These divisions are reminiscent of the groupings we proposed in Paper III based on MDF shapes. We classified Fornax, Leo I, and Leo II as "infall-dominated" and Sextans, Draco, Canes Venatici I, and Ursa Minor as "outflowdominated." Sculptor sat in its own class. The similar groupings based on MDF and $[\alpha / \mathrm{Fe}]$ unsurprisingly reaffirm that the SFH shapes both the MDF and the element ratio distributions.

The MW satellite galaxies more luminous than Fornax sample a regime of greater integrated star formation and higher metallicity. Pompéia et al. (2008) measured $[\alpha / \mathrm{Fe}]$ for individual red giants in the disk of the Large Magellanic Cloud (LMC), and Mucciarelli et al. (2008) measured the same for red giants in LMC GCs. The stars span the range $-1.2 \leqslant[\mathrm{Fe} / \mathrm{H}] \leqslant$
-0.3 with one additional star at $[\mathrm{Fe} / \mathrm{H}]=-1.7$. The $[\mathrm{Ca} / \mathrm{Fe}]$ ratios of the disk stars decline slightly with increasing $[\mathrm{Fe} / \mathrm{H}]$, but the other element ratios are nearly flat. In fact, the LMC stars seem to follow the same $[\alpha / \mathrm{Fe}]$ trends as Fornax or Leo I, albeit shifted to higher $[\mathrm{Fe} / \mathrm{H}]$, except for $[\mathrm{Ti} / \mathrm{Fe}]$. The average $[\mathrm{Ti} / \mathrm{Fe}]$ in the LMC is about 0.1 dex higher than Leo I and $0.3 \mathrm{dex}$ higher than Fornax. The Sagittarius dSph also shows a higher average [Ti/Fe] than Fornax or Leo I (Chou et al. 2010). Also, $[\mathrm{Ti} / \mathrm{Fe}]$ in Sagittarius declines with increasing $[\mathrm{Fe} / \mathrm{H}]$ over the entire range that Chou et al. sampled $(-1.5 \leqslant[\mathrm{Fe} / \mathrm{H}] \leqslant+0.1)$.

The available evidence indicates that the evolution of $[\alpha / \mathrm{Fe}]$ with $[\mathrm{Fe} / \mathrm{H}]$ is nearly universal in MW satellite galaxies except for $[\mathrm{Ti} / \mathrm{Fe}]$ at $[\mathrm{Fe} / \mathrm{H}] \gtrsim-1.3$. The average values of $[\mathrm{Ti} / \mathrm{Fe}]$ for the dSphs and the LMC at these metallicities vary from about -0.3 (Sculptor) to 0.0 (LMC and Sagittarius), and the slopes vary from $\Delta[\mathrm{Ti} / \mathrm{Fe}] / \Delta[\mathrm{Fe} / \mathrm{H}] \approx-0.8$ (Sagittarius) to 0.0 (Fornax). Ti is both an $\alpha$ element and an iron-group element, and it has an appreciable yield from both Types II and Ia SNe (Woosley \& Weaver 1995). Therefore, $[\mathrm{Ti} / \mathrm{Fe}]$ responds to changes in the SFR and the IMF differently from the "purer" $\alpha$ elements, like Mg and Si. Unfortunately, our chemical evolution model failed to reproduce realistic values of $[\mathrm{Ti} / \mathrm{Fe}]$ because the theoretical Type II SN yields of Ti were too small. We suggest that future work explore ratios such as $[\mathrm{Mg} / \mathrm{Ti}]$ to better understand why $[\mathrm{Ti} / \mathrm{Fe}]$ behaves differently in different dwarf galaxies at high $[\mathrm{Fe} / \mathrm{H}]$.

$$
\text { 5.1.2. }[\mathrm{Mg} / \mathrm{Fe}]
$$

Our data set for the first time has enabled the exploration of the bulk properties of $[\alpha / \mathrm{Fe}]$ in $\mathrm{dSphs}$ that span two orders of magnitude in luminosity. In particular, Figure 13 shows that $[\mathrm{Mg} / \mathrm{Fe}]$ values higher than in the $\mathrm{MW}$ are not unique to the extremely metal-poor stars in dSphs (e.g., Frebel et al. 2010 b) but also exist in stars of more modest metallicity $([\mathrm{Fe} / \mathrm{H}] \lesssim-1.8)$.

Factors beyond the SFH may affect the absolute value of $[\mathrm{Mg} / \mathrm{Fe}]$ and other element ratios at low metallicity. First, changing the IMF alters $[\alpha / \mathrm{Fe}]$ because Type II SN yields depend on the mass of the exploding star. Second, the early gas mass of the dSph might change the shape of the low-metallicity $[\alpha / \mathrm{Fe}]$ distribution also because $\mathrm{SN}$ yields depend on mass. The first $\mathrm{SNe}$ in a galaxy can more efficiently enrich a small gas mass than a large gas mass. Massive SNe explode before less massive $\mathrm{SNe}$, and massive $\mathrm{SNe}$ generally produce higher $[\alpha / \mathrm{Fe}]$. As a result, $[\alpha / \mathrm{Fe}]$ at low metallicity could depend on the initial gas mass that was enriched by the first SNe. This effect possibly explains the larger $[\mathrm{Mg} / \mathrm{Fe}]$ in dSphs than in the MW. We suggest that the stars at $[\mathrm{Fe} / \mathrm{H}] \sim-2.5$ in $\mathrm{dSphs}$ were enriched by $\mathrm{SNe}$ of higher average mass than the stars at $[\mathrm{Fe} / \mathrm{H}] \sim-2$ in the MW. Finally, the shape of the abundance distribution might depend on the early gas mass because SN yields also depend on metallicity. In addition to sampling higher mass $\mathrm{SNe}$, stars at a given $[\mathrm{Fe} / \mathrm{H}]$ in a lower mass galaxy sample lower metallicity $\mathrm{SNe}$ than stars at the same $[\mathrm{Fe} / \mathrm{H}]$ in a higher mass galaxy.

\subsubsection{Unexplained Details}

Many details in Figure 13 defy obvious explanations. For example, the $[\mathrm{Ca} / \mathrm{Fe}]$ ratio is flatter than the other element ratios. Sculptor has a strangely large $[\mathrm{Ca} / \mathrm{Fe}]$ at low $[\mathrm{Fe} / \mathrm{H}]$. The [Si/Fe] trend for Fornax is above the other dSphs' trends, but the other element ratios seem consistent. Similarly, the 


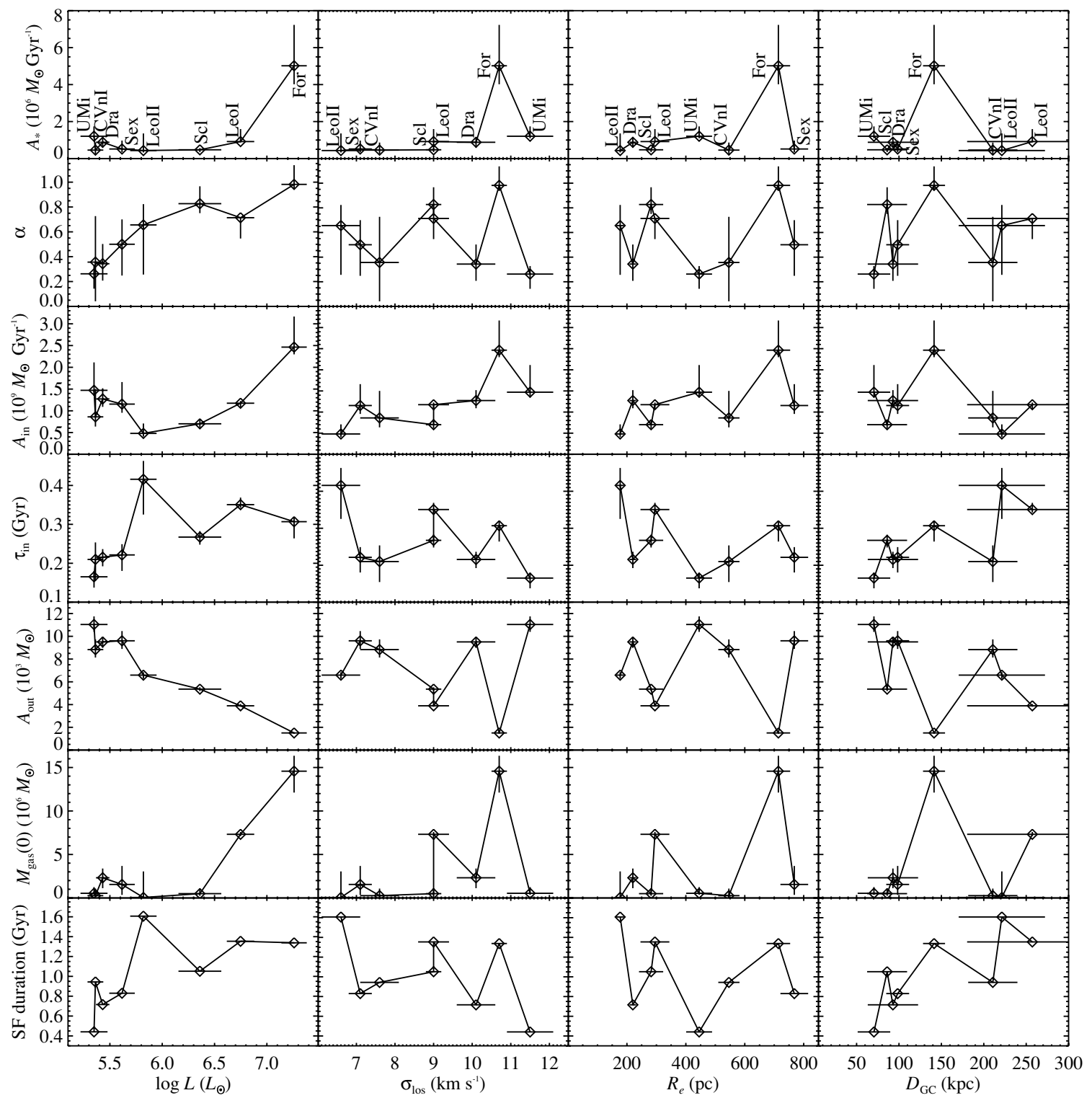

Figure 14. Trends of the best-fit chemical evolution models with galaxy properties: luminosity (Irwin \& Hatzidimitriou 1995; Martin et al. 2008a), line-of-sight velocity dispersion, two-dimensional projected half-light radius (both from Wolf et al. 2010 and references therein), and Galactocentric distance. From top to bottom, the parameters are the SFR normalization, SFR exponent (Equation (5)), gas infall rate, gas infall timescale (Equation (14)), gas expelled per SN (Equation (15)), and initial gas mass (Equation (16)). Table 2 gives the same data. The bottom row also shows the duration of star formation (see Table 3), which is a quantity derived from the model, not a free parameter. The only galaxy property to show a trend is luminosity.

$[\mathrm{Ti} / \mathrm{Fe}]$ ratio-and only $[\mathrm{Ti} / \mathrm{Fe}]$ - for Leo I lies above the other dSphs. Ursa Minor, despite being the least luminous dSph in the figure, has the second largest $[\alpha / \mathrm{Fe}]$ at a given metallicity for much of the metallicity range. The slope of $[\mathrm{Mg} / \mathrm{Fe}]$ flattens for all of the dSphs at $[\mathrm{Fe} / \mathrm{H}] \gtrsim-1.2$, but the slope of $[\mathrm{Si} / \mathrm{Fe}]$ flattens only for Fornax and Leo II.

We suggest that future work examine the abundance catalog in more detail. For example, element ratios with a denominator other than Fe could constrain the IMF. The predicted yields of $[\mathrm{Mg} / \mathrm{Si}]$ decrease from +0.2 for a progenitor mass of $18 M_{\odot}$ to -0.3 for a progenitor mass of $40 M_{\odot}$ (Nomoto et al. 2006). Our data set possesses the sample size and precision to address such questions.

\subsection{Trends in Chemical Evolution Model Parameters}

We now invoke the best-fit parameters of the chemical evolution model in a more quantitative discussion of the correlation between abundance distributions and galaxy properties. Figure 14 presents the parameters against luminosity, line-of-sight velocity dispersion, half-light radius, and Galactocentric distance. In addition to the model parameters, the bottom row of the figure shows the star formation duration, which is a quantity derived from the best-fit model, not a free parameter.

Luminosity can reasonably be expected to show the best correlation with quantities related to SF. Of the four abscissas in Figure 14, $L$ is the only one that could be predicted from our simple chemical evolution model. Roughly, $L$ is the integral of past SF, modulated by the reddening and dimming associated with aging. Therefore, it is not surprising that the chemical evolution parameters vary with $L$. Although we have plotted the SF parameters against $L, L$ is not necessarily the independent variable. Luminosity is a present-day quantity, and the stars did not know the final stellar mass of the galaxy while they were forming. The SFH determines the present luminosity. 


\subsubsection{Star Formation Rate Parameters}

The SFR normalization, $A_{*}$, is roughly constant at $\sim 5 \times$ $10^{5} M_{\odot} \mathrm{Gyr}^{-1}$ for galaxies less luminous than Leo I. The value roughly doubles for Leo I and increases by an order of magnitude for Fornax. The increase in $A_{*}$ is expected because a more luminous galaxy must have formed more stars than a less luminous galaxy. If the SF timescale does not change much with luminosity, then the SFR must. We observe that the SF duration changes by a factor of about four across the luminosity range. Therefore, we estimate a range of 40 in luminosity. The actual $L$ range is 80 , but our simple estimate ignored the ages of the stellar population and the other model parameters which affect the SFR, such as $\tau_{\text {in }}$.

The exponent of the SFR law, $\alpha$, also varies with $L$. If we assume that SFR is proportional to gas volume density, then $\alpha$ may indicate the degree to which the gas was concentrated in the center of the galaxy. However, we find no correlation between $\alpha$ and the concentration of the light profiles (Irwin \& Hatzidimitriou 1995; not shown in Figure 14). Our interpretation of $\alpha$ is purely speculative because SF is a complex process affected by many external factors, such as an ionizing radiation background. These factors become more difficult to predict for smaller galaxies (e.g., Gnedin \& Kravtsov 2010).

\subsubsection{Gas Infall Parameters}

The intensity of infalling gas (or gas cooling to become available for SF) drives the SFR. The parameter $A_{\text {in }}$ is closely related to $A_{*}$. The dSph cannot maintain a high SFR without the addition of new gas. Therefore, a luminous galaxy must have had large values of both $A_{*}$ and $A_{\text {in }}$. Alternatively, a luminous galaxy could have started its life with a large reservoir of gas. However, in order to prevent too many metal-poor stars from forming early, new gas must have been added during the SF lifetime. The net result is that $A_{*}, A_{\text {in }}$, and $M_{\text {gas }}(0)$ are highly covariant.

The most likely timescales for gas infall (or cooling) vary from 0.17 to 0.42 Gyr. It may be significant that none of the timescales exceeds 0.42 Gyr. We propose three conjectures. First, $\tau_{\text {in }}$ may reflect the time the $\mathrm{dSph}$ requires to accumulate gas. The central densities of dSphs are similar (Mateo 1998; Gilmore et al. 2007; Strigari et al. 2008). Therefore, the similar gravitational potentials of the $\mathrm{dSphs}$ themselves might enforce similarly small gas accretion timescales.

Second, the dSphs' environment may set the $\tau_{\text {in }}$ timescale. Interestingly, $\sim 0.1 \mathrm{Gyr}$ was the timescale for the Galaxy's monolithic collapse proposed by Eggen et al. (1962). This collapse time corresponds to a period when the gas in the vicinity of the MW was rapidly coalescing into individual structures, such as the proto-Galaxy and the dSphs. After $0.1 \mathrm{Gyr}$, gas accretion would have declined considerably because the MW and its satellites would by then have accreted the bulk of the surrounding gas. In the $\Lambda \mathrm{CDM}$ paradigm, the formation time for a dSph-sized dark matter halo is only 0.4 Gyr after the Big Bang (Wechsler et al. 2002). Therefore, our most likely gas accretion timescales are consistent with both cosmogonies.

Third, the time from the formation of the first stars to cosmological reionization is roughly 0.5 Gyr. Ricotti \& Gnedin (2005) referred to all eight of our dSphs as "true" or "polluted fossils," meaning that all or most of their stars formed before reionization. Our models are sensitive to the bulk of the population, and not the few younger stars present in most $\mathrm{dSphs}$. Therefore, the best-fit values of $\tau_{\text {in }}$ may be probing the pre-reionization SF timescale. Fornax must be a exception because the bulk of its population formed after reionization. The majority stellar populations in other dSphs may be fossils with SF timescales on the order of the reionization time. The (small) dispersion among our $\tau_{\text {in }}$ values may be a result of temporally protracted, spatially inhomogeneous reionization (Miralda-Escudé et al. 2000). However, we note that our derived SF durations are longer than 0.5 Gyr except for Ursa Minor. To the extent that these durations are accurate, we surmise that reionization is one of several mechanisms that inhibited SF in dSphs.

\subsubsection{Supernova Winds}

The role of $\mathrm{SN}$ feedback for dSphs has been emphasized repeatedly. Dekel \& Silk (1986) posited that SN feedback regulates the SFR for dwarf galaxies. It can cause a terminal wind, or it can blow out gas that is later re-accreted. For the smallest galaxies, including the dSphs presented here, radiation feedback also plays a significant role (Dekel \& Woo 2003). The best-fit $\mathrm{SN}$ wind intensities, $A_{\text {out }}$, also show a strong correlation with $L$. More luminous dSphs experienced more intense winds. This trend is a direct result of the metallicity-luminosity relation for dSphs (e.g., Paper III). For reasons discussed in Paper III, more intense gas outflow lowers the effective metal yield. Therefore, the less luminous, more metal-poor dSphs naturally show more gas outflow. However, we expected that $A_{\text {out }}$ also correlate with the velocity dispersion, a measure of the depth of the potential well. No such correlation exists. The lack of correlation is puzzling, but the gas blowout depends on the unmeasurable mass density profile at the time of SF and on the locations of the SNe within the gravitational potential.

\subsubsection{Galaxy Properties Other than Luminosity}

The model parameters are insensitive to galaxy properties other than $L$. The velocity dispersions of dSphs do not span nearly as large a range as their luminosities, which may partly explain the lack of dependence on $\sigma_{\text {los }}$. The half-light radius and luminosity together are related to the galaxy's surface brightness and stellar density. It does not seem that the SF parameters in our model depend significantly on these quantities. The timescales, $\tau_{\text {in }}$, and the SF duration, may depend weakly on Galactocentric distance. The Pearson linear correlation coefficient between $\tau_{\text {in }}$ and $D_{\mathrm{GC}}$ is 0.69 . Because $\tau_{\text {in }}$ basically represents the SF duration (the correlation coefficient between $\tau_{\text {in }}$ and the SF duration is 0.96), this relation may indicate that more distant dSphs survive SF-truncating interactions with the MW longer than closer dSphs. In fact, Silk et al. (1987) suggested that host galaxies competed with their satellites for gas accretion. The more distant satellites, such as dwarf irregulars, successfully accreted more gas to power present star formation than the closer satellites, such as dSphs. Orbital history would be a better indicator of past interaction with the MW. Orbital parameters based on proper motions are available for Fornax (Piatek et al. 2007), Sculptor (Piatek et al. 2006), and Ursa Minor (Piatek et al. 2005). Sohn et al. (2007) also constrained the orbit of Leo I based on the shape and dynamics of tidal debris. We leave orbital analyses for future work.

We conclude that luminosity is more directly related to a dSph's SFH than dynamical or morphological properties. The present luminosity cannot drive the past star formation, but the luminosity does mirror a single parameter which determines the SFH. This conclusion is similar to the fundamental line for dwarf galaxies defined by Woo et al. (2008). They also found that 
stellar mass (closely related to luminosity) is the best predictor of other dSph properties. However, stellar mass loss by tidal stripping may obfuscate the correlation between present stellar mass and past star formation.

\section{SUMMARY AND CONCLUSIONS}

We have made a first attempt at quantitative chemical evolution models for the large sample of multi-element abundance measurements for MW dSphs that we published in Paper II. Our simple model is a significant improvement to the analytical models of the metallicity distributions that we explored in Paper III. We fit the MDF and $[\alpha / \mathrm{Fe}]$ distribution simultaneously to derive the SF and gas flow histories of each of eight dSphs spanning about two orders of magnitude in luminosity. Our model produces reasonable fits to the abundance distributions of dSphs whose CMDs show that most or all of their stars are older than 10 Gyr.

We draw the following conclusions from our models and from the general trends in abundance distributions (Figure 13).

1. The $[\alpha / \mathrm{Fe}]$ ratios evolve with metallicity along nearly the same path for all dSphs. The average value of $[\mathrm{Mg} / \mathrm{Fe}],[\mathrm{Si} / \mathrm{Fe}],[\mathrm{Ca} / \mathrm{Fe}]$, and $[\mathrm{Ti} / \mathrm{Fe}]$ drops from +0.4 at $[\mathrm{Fe} / \mathrm{H}]=-2.5$ to 0.0 at $[\mathrm{Fe} / \mathrm{H}] \approx-1.2$, where the slope flattens.

2. No low-metallicity plateaus or knees exist in $[\alpha / \mathrm{Fe}]$ versus $[\mathrm{Fe} / \mathrm{H}]$ space for any $\mathrm{dSph}$ at $[\mathrm{Fe} / \mathrm{H}]>-2.5$. We conclude that Type Ia SNe contributed to chemical evolution for all but the most metal-poor stars.

3. The $[\mathrm{Mg} / \mathrm{Fe}]$ ratio in $\mathrm{dSphs}$ exceeds that of the $\mathrm{MW}$ at $[\mathrm{Fe} / \mathrm{H}] \lesssim-1.8$. We suggest that the abundance ratios of stars in low-mass systems are more sensitive to the mass and metallicity dependence of Type II SNe yields than stars at the same metallicity in higher-mass systems, such as the progenitors of the inner MW halo.

4. The dSphs may be grouped based on their $[\alpha / \mathrm{Fe}]$ distributions into roughly the same groups that we defined based on their metallicity distributions (Paper III). The more luminous dSphs have infall-dominated MDFs and slightly higher $\langle[\alpha / \mathrm{Fe}]\rangle$ at a given $[\mathrm{Fe} / \mathrm{H}]$. The less luminous $\mathrm{dSphs}$ have outflow-dominated MDFs and slightly lower $\langle[\alpha / \mathrm{Fe}]\rangle$ at the same $[\mathrm{Fe} / \mathrm{H}]$.

5. Some SF model parameters correlate with present luminosity, but not with velocity dispersion, half-light radius, or Galactocentric distance except for a possible correlation between gas infall timescale and $D_{\mathrm{GC}}$.

6. The gas flow histories for all dSphs except Fornax are characterized by large amounts of gas loss, probably driven by $\mathrm{SN}$ winds. Less luminous dSphs experienced more intense gas loss.

7. Allowing SN winds to be metal-enhanced drastically reduces the amount of gas infall and outflow required to explain the observed abundance distributions.

8. The gas infall timescale does not exceed 0.42 Gyr. This possibly reflects the amount of time ancient stars had to form before reionization ended star formation.

9. The derived star formation timescales are extremely sensitive to the delay time for the first Type Ia SN. Increasing the delay time from $0.1 \mathrm{Gyr}$ to $0.3 \mathrm{Gyr}$ results in a star formation duration in Sculptor inflated by a factor of 3.5.

10. The presence of bumps in the MDFs and stars with $[\alpha / \mathrm{Fe}]$ ratios far from the average trend lines suggests that the SFHs of dSphs were characterized by bursts, which are not included in our model. Bursts are a common feature of more sophisticated models.

Some of our conclusions (5-10) depend on the realism of our chemical evolution model. Many more sophisticated models exist, and we encourage their application to our data set. Paper II contains the complete abundance catalog.

The major strength of the present work is that we apply the same model to a homogeneous data set of hundreds of stars in each of eight dSphs. The sample size and diversity of galaxies has allowed us to present an overview of chemical evolution in dwarf galaxies. We have discovered patterns not apparent in previous data sets due to small samples or lack of diversity among the well-sampled galaxies. In particular, we have shown that $[\alpha / \mathrm{Fe}]$ distributions of $\mathrm{dSphs}$ do not form a sequence of knees corresponding to the metallicities at which Type Ia $\mathrm{SNe}$ began to explode. Instead, the $[\alpha / \mathrm{Fe}]$ patterns of all $\mathrm{dSphs}$ are largely the same, but different $\mathrm{dSphs}$ sample different regions in metallicity.

We thank John Johnson, Hai Fu, Julianne Dalcanton, Chris Sneden, and Bob Kraft for insightful discussions. Support for this work was provided by NASA through Hubble Fellowship grant 51256.01 awarded to E.N.K. by the Space Telescope Science Institute, which is operated by the Association of Universities for Research in Astronomy, Inc., for NASA, under contract NAS 5-26555. S.R.M. acknowledges support from NSF grants AST-0307851 and AST-0807945, and from the SIM Lite key project "Taking Measure of the Milky Way" under NASA/ JPL contract 1228235. P.G. acknowledges NSF grants AST0507483, AST-0607852, and AST-0808133.

The authors recognize and acknowledge the very significant cultural role and reverence that the summit of Mauna Kea has always had within the indigenous Hawaiian community. We are most fortunate to have the opportunity to conduct observations from this mountain.

\section{Facility: Keck:II (DEIMOS)}

\section{REFERENCES}

Aaronson, M., Hodge, P. W., \& Olszewski, E. W. 1983, ApJ, 267, 271

Aaronson, M., Liebert, J., \& Stocke, J. 1982, ApJ, 254, 507

Aaronson, M., \& Mould, J. 1980, ApJ, 240, 804

Abia, C. 2008, AJ, 136, 250

Anders, E., \& Grevesse, N. 1989, Geochim. Cosmochim. Acta, 53, 197

Aparicio, A., Carrera, R., \& Martínez-Delgado, D. 2001, AJ, 122, 2524

Azzopardi, M., Lequeux, J., \& Westerlund, B. E. 1985, A\&A, 144, 388

Babusiaux, C., Gilmore, G., \& Irwin, M. 2005, MNRAS, 359, 985

Battaglia, G., Helmi, A., Tolstoy, E., Irwin, M., Hill, V., \& Jablonka, P. 2008, ApJ, 681, L13

Battaglia, G., et al. 2006, A\&A, 459, 423

Bell, R. A. 1985, PASP, 97, 219

Bellazzini, M., Ferraro, F. R., Origlia, L., Pancino, E., Monaco, L., \& Oliva, E. 2002, AJ, 124, 3222

Bellazzini, M., Ferraro, F. R., \& Pancino, E. 2001, MNRAS, 327, L15

Bensby, T., Feltzing, S., \& Lundström, I. 2003, A\&A, 410, 527

Bernard, E. J., et al. 2009, ApJ, 699, 1742

Bosler, T. L., Smecker-Hane, T. A., \& Stetson, P. B. 2007, MNRAS, 378, 318

Bullock, J. S., \& Johnston, K. V. 2005, ApJ, 635, 931

Bullock, J. S., Kravtsov, A. V., \& Weinberg, D. H. 2000, ApJ, 539, 517

Buonanno, R., Corsi, C. E., Castellani, M., Marconi, G., Fusi Pecci, F., \& Zinn, R. 1999, AJ, 118, 1671

Burris, D. L., Pilachowski, C. A., Armandroff, T. E., Sneden, C., Cowan, J. J., \& Roe, H. 2000, ApJ, 544, 302

Caputo, F., Cassisi, S., Castellani, M., Marconi, G., \& Santolamazza, P. 1999, AJ, 117,2199

Carigi, L., \& Hernandez, X. 2008, MNRAS, 390, 582

Carrera, R., Aparicio, A., Martínez-Delgado, D., \& Alonso-García, J. 2002, AJ, 123,3199 
Chou, M.-Y., Cunha, K., Majewski, S. R., Smith, V. V., Patterson, R. J., MartínezDelgado, D., \& Geisler, D. 2010, ApJ, 708, 1290

Cohen, J. G., \& Huang, W. 2009, ApJ, 701, 1053

Cohen, J. G., \& Huang, W. 2010, ApJ, 719, 931

Coleman, M. G., \& de Jong, J. T. A. 2008, ApJ, 685, 933

Colucci, J. E., Bernstein, R. A., Cameron, S., McWilliam, A., \& Cohen, J. G. 2009, ApJ, 704, 385

Cudworth, K. M., Olszewski, E. W., \& Schommer, R. A. 1986, AJ, 92, 766

Da Costa, G. S. 1984, ApJ, 285, 483

Dalcanton, J. J., et al. 2009, ApJS, 183, 67

Dekel, A., \& Silk, J. 1986, ApJ, 303, 39

Dekel, A., \& Woo, J. 2003, MNRAS, 344, 1131

Demarque, P., \& Hirshfeld, A. W. 1975, ApJ, 202, 346

Demers, S., Kunkel, W. E., \& Hardy, E. 1979, ApJ, 232, 84

Diemand, J., Kuhlen, M., \& Madau, P. 2007, ApJ, 667, 859

Dolphin, A. E., Weisz, D. R., Skillman, E. D., \& Holtzman, J. A. 2005 , in Resolved Stellar Populations, ed. D. Valls-Gabaud \& M. Chavez, arXiv:astro-ph/0506430

Edvardsson, B., Andersen, J., Gustafsson, B., Lambert, D. L., Nissen, P. E., \& Tomkin, J. 1993, A\&A, 275, 101

Eggen, O. J., Lynden-Bell, D., \& Sandage, A. R. 1962, ApJ, 136, 748

Faria, D., Feltzing, S., Lundström, I., Gilmore, G., Wahlgren, G. M., Ardeberg, A., \& Linde, P. 2007, A\&A, 465, 357

Fenner, Y., Gibson, B. K., Gallino, R., \& Lugaro, M. 2006, ApJ, 646, 184

François, P., Matteucci, F., Cayrel, R., Spite, M., Spite, F., \& Chiappini, C. 2004, A\&A, 421, 613

Frebel, A., Kirby, E. N., \& Simon, J. D. 2010a, Nature, 464, 72

Frebel, A., Simon, J. D., Geha, M., \& Willman, B. 2010b, ApJ, 708, 560

Fulbright, J. P. 2000, AJ, 120, 1841

Fulbright, J. P. 2002, AJ, 123, 404

Galama, T. J., et al. 1998, Nature, 395, 670

Gallart, C., Freedman, W. L., Aparicio, A., Bertelli, G., \& Chiosi, C. 1999a, AJ, 118,2245

Gallart, C., et al. 1999b, ApJ, 514, 665

Geha, M., Willman, B., Simon, J. D., Strigari, L. E., Kirby, E. N., Law, D. R., \& Strader, J. 2009, ApJ, 692, 1464

Geisler, D., Smith, V. V., Wallerstein, G., Gonzalez, G., \& Charbonnel, C. 2005, AJ, 129, 1428

Gilmore, G., Wilkinson, M. I., Wyse, R. F. G., Kleyna, J. T., Koch, A., Evans, N. W., \& Grebel, E. K. 2007, ApJ, 663, 948

Gilmore, G., \& Wyse, R. F. G. 1991, ApJ, 367, L55

Gnedin, N. Y., \& Kravtsov, A. V. 2010, ApJ, submitted (arXiv:1004.0003)

Governato, F., Willman, B., Mayer, L., Brooks, A., Stinson, G., Valenzuela, O., Wadsley, J., \& Quinn, T. 2007, MNRAS, 374, 1479

Gratton, R. G., \& Sneden, C. 1988, A\&A, 204, 193

Gratton, R. G., \& Sneden, C. 1991, A\&A, 241, 501

Gratton, R. G., \& Sneden, C. 1994, A\&A, 287, 927

Graves, G. J., \& Schiavon, R. P. 2008, ApJS, 177, 446

Grebel, E. K., \& Stetson, P. B. 1999, in IAU Symp., Vol. 192, The Stellar Content of Local Group Galaxies, ed. P. Whitelock \& R. Cannon (San Francisco, CA: ASP), 165

Grillmair, C. J., et al. 1998, AJ, 115, 144

Gullieuszik, M., Held, E. V., Rizzi, L., Saviane, I., Momany, Y., \& Ortolani, S. 2007, A\&A, 467, 1025

Gullieuszik, M., Held, E. V., Saviane, I., \& Rizzi, L. 2009, A\&A, 500, 735

Hanson, R. B., Sneden, C., Kraft, R. P., \& Fulbright, J. 1998, AJ, 116, 1286

Held, E. V., Clementini, G., Rizzi, L., Momany, Y., Saviane, I., \& Di Fabrizio, L. 2001, ApJ, 562, L39

Held, E. V., Saviane, I., Momany, Y., \& Carraro, G. 2000, ApJ, 530, L85

Hirshfeld, A. W. 1980, ApJ, 241, 111

Holtzman, J. A., Afonso, C., \& Dolphin, A. 2006, ApJS, 166, 534

Howell, D. A., et al. 2009, ApJ, 691, 661

Hurley-Keller, D., Mateo, M., \& Nemec, J. 1998, AJ, 115, 1840

Ikuta, C., \& Arimoto, N. 2002, A\&A, 391, 55

Irwin, M., \& Hatzidimitriou, D. 1995, MNRAS, 277, 1354

Ivans, I. I., Sneden, C., James, C. R., Preston, G. W., Fulbright, J. P., Höflich, P. A., Carney, B. W., \& Wheeler, J. C. 2003, ApJ, 592, 906

Iwamoto, K., Brachwitz, F., Nomoto, K., Kishimoto, N., Umeda, H., Hix, W. R., \& Thielemann, F.-K. 1999, ApJS, 125, 439

Iwamoto, K., et al. 1998, Nature, 395, 672

Johnson, J. A. 2002, ApJS, 139, 219

Johnston, K. V., Bullock, J. S., Sharma, S., Font, A., Robertson, B. E., \& Leitner, S. N. 2008, ApJ, 689, 936

Karakas, A. I. 2010, MNRAS, 403, 1413

Kennicutt, R. C., Jr. 1998, ApJ, 498, 541

Kinman, T. D., \& Kraft, R. P. 1980, AJ, 85, 415
Kinman, T. D., Kraft, R. P., \& Suntzeff, N. B. 1981, in Physical Processes in Red Giants, ed. I. Iben \& A. Renzini (Dordrecht: Reidel), 71

Kirby, E. N., Guhathakurta, P., Bolte, M., Sneden, C., \& Geha, M. C. 2009, ApJ, 705, 328 (Paper I)

Kirby, E. N., Lanfranchi, G. A., Simon, J. D., Cohen, J. G., \& Guhathakurta, P. 2010a, ApJ, 727, 78 (Paper III)

Kirby, E. N., Simon, J. D., Geha, M., Guhathakurta, P., \& Frebel, A. 2008, ApJ, $685, \mathrm{~L} 43$

Kirby, E. N., et al. 2010b, ApJS, 191, 352 (Paper II)

Kobayashi, C., \& Nomoto, K. 2009, ApJ, 707, 1466

Kobayashi, C., Tsujimoto, T., Nomoto, K., Hachisu, I., \& Kato, M. 1998, ApJ, 503, L155

Koch, A., Grebel, E. K., Wyse, R. F. G., Kleyna, J. T., Wilkinson, M. I., Harbeck, D. R., Gilmore, G. F., \& Evans, N. W. 2006, AJ, 131, 895

Kodama, T. 1997, PhD thesis,Univ. of Tokyo

Kroupa, P., Tout, C. A., \& Gilmore, G. 1993, MNRAS, 262, 545

Kuehn, C., et al. 2008, ApJ, 674, L81

Lanfranchi, G. A., \& Matteucci, F. 2003, MNRAS, 345, 71

Lanfranchi, G. A., \& Matteucci, F. 2004, MNRAS, 351, 1338

Lanfranchi, G. A., \& Matteucci, F. 2007, A\&A, 468, 927

Lanfranchi, G. A., \& Matteucci, F. 2010, A\&A, 512, A85

Lanfranchi, G. A., Matteucci, F., \& Cescutti, G. 2006, A\&A, 453, 67

Lanfranchi, G. A., Matteucci, F., \& Cescutti, G. 2008, A\&A, 481, 635

Larson, D., et al. 2010, ApJS, in press (arXiv:1001.4635)

Larson, R. B. 1972, Nature, 236, 7

Larson, R. B. 1974, MNRAS, 169, 229

Lee, M. G., Freedman, W., Mateo, M., Thompson, I., Roth, M., \& Ruiz, M.-T. 1993, AJ, 106, 1420

Lee, M. G., Yuk, I.-S., Park, H. S., Harris, J., \& Zaritsky, D. 2009, ApJ, 703, 692

Lehnert, M. D., Bell, R. A., Hesser, J. E., \& Oke, J. E. 1992, ApJ, 395, 466

Letarte, B., et al. 2010, A\&A, 523, A17

Lin, D. N. C., \& Faber, S. M. 1983, ApJ, 266, L21

Lynden-Bell, D. 1975, Vistas Astron., 19, 299

Mac Low, M.-M., \& Ferrara, A. 1999, ApJ, 513, 142

Maeder, A., \& Meynet, G. 1989, A\&A, 210, 155

Majewski, S. R., Ostheimer, J. C., Kunkel, W. E., \& Patterson, R. J. 2000a, AJ, 120,2550

Majewski, S. R., Ostheimer, J. C., Patterson, R. J., Kunkel, W. E., Johnston, K. V., \& Geisler, D. 2000b, AJ, 119, 760

Majewski, S. R., Siegel, M. H., Patterson, R. J., \& Rood, R. T. 1999, ApJ, 520, L33

Majewski, S. R., et al. 2002, in ASP Conf. Ser. 285, Modes of Star Formation and the Origin of Field Populations, ed. E. Grebel \& W. Brandner (San Francisco, CA: ASP), 199

Maoz, D., Sharon, K., \& Gal-Yam, A. 2010, ApJ, 722, 1879

Mapelli, M., Ripamonti, E., Battaglia, G., Tolstoy, E., Irwin, M. J., Moore, B., \& Sigurdsson, S. 2009, MNRAS, 396, 1771

Marcolini, A., D'Ercole, A., Battaglia, G., \& Gibson, B. K. 2008, MNRAS, 386,2173

Marcolini, A., D’Ercole, A., Brighenti, F., \& Recchi, S. 2006, MNRAS, 371, 643

Marigo, P., \& Girardi, L. 2007, A\&A, 469, 239

Martin, N. F., de Jong, J. T. A., \& Rix, H.-W. 2008a, ApJ, 684, 1075

Martin, N. F., et al. 2008b, ApJ, 672, L13

Martínez-Delgado, D., Alonso-García, J., Aparicio, A., \& Gómez-Flechoso, M. A. 2001, ApJ, 549, L63

Mateo, M. L. 1998, ARA\&A, 36, 435

Mateo, M., Olszewski, E. W., \& Walker, M. G. 2008, ApJ, 675, 201

Mathews, W. G., \& Baker, J. C. 1971, ApJ, 170, 241

Matteucci, F. 2008, Lectures for the 37th Saas-Fee Advanced Course, (arXiv:0804.1492)

Matteucci, F., \& Brocato, E. 1990, ApJ, 365, 539

Matteucci, F., Spitoni, E., Recchi, S., \& Valiante, R. 2009, A\&A, 501, 531

McWilliam, A. 1998, AJ, 115, 1640

McWilliam, A., \& Bernstein, R. A. 2008, ApJ, 684, 326

McWilliam, A., Preston, G. W., Sneden, C., \& Shectman, S. 1995, AJ, 109, 2736

McWilliam, A., \& Smecker-Hane, T. A. 2005a, ApJ, 622, L29

McWilliam, A., \& Smecker-Hane, T. A. 2005b, in ASP Conf. Ser. 336, Cosmic Abundances as Records of Stellar Evolution and Nucleosynthesis in honor of D. L. Lambert, ed. T. G. Barnes III \& F. N. Bash (San Francisco, CA: ASP), 221

Mighell, K. J. 1990, A\&AS, 82, 1

Mighell, K. J. 1997, AJ, 114, 1458

Mighell, K. J., \& Burke, C. J. 1999, AJ, 118, 366

Mighell, K. J., \& Rich, R. M. 1996, AJ, 111, 777 
Miralda-Escudé, J., Haehnelt, M., \& Rees, M. J. 2000, ApJ, 530, 1

Monkiewicz, J., et al. 1999, PASP, 111, 1392

Mori, M., Ferrara, A., \& Madau, P. 2002, ApJ, 571, 40

Mucciarelli, A., Carretta, E., Origlia, L., \& Ferraro, F. R. 2008, AJ, 136, 375

Muñoz, R. R., et al. 2005, ApJ, 631, L137

Muñoz, R. R., et al. 2006, ApJ, 649, 201

Napolitano, N. R., Romanowsky, A. J., \& Tortora, C. 2010, MNRAS, 405, 2351

Nissen, P. E., \& Schuster, W. J. 1997, A\&A, 326, 751

Nomoto, K., Tominaga, N., Umeda, H., Kobayashi, C., \& Maeda, K. 2006, Nucl. Phys. A, 777, 424

Norris, J., \& Bessell, M. S. 1978, ApJ, 225, L49

Norris, J. E., Gilmore, G., Wyse, R. F. G., Wilkinson, M. I., Belokurov, V., Evans, N. W., \& Zucker, D. B. 2008, ApJ, 689, L113

Norris, J. E., Wyse, R. F. G., Gilmore, G., Yong, D., Frebel, A., Wilkinson, M. I., Belokurov, V., \& Zucker, D. B. 2010a, ApJ, 723, 1632

Norris, J. E., Yong, D., Gilmore, G., \& Wyse, R. F. G. 2010b, ApJ, 711, 350

Norris, J., \& Zinn, R. 1975, ApJ, 202, 335

Olszewski, E. W., \& Aaronson, M. 1985, AJ, 90, 2221

Orban, C., Gnedin, O. Y., Weisz, D. R., Skillman, E. D., Dolphin, A. E., \& Holtzman, J. A. 2008, ApJ, 686, 1030

Padovani, P., \& Matteucci, F. 1993, ApJ, 416, 26

Pagel, B. E. J. 1997, Nucleosynthesis and Chemical Evolution of Galaxies (Cambridge: Cambridge Univ. Press)

Pagel, B. E. J., \& Tautvaišienè, G. 1995, MNRAS, 276, 505

Piatek, S., Pryor, C., Bristow, P., Olszewski, E. W., Harris, H. C., Mateo, M., Minniti, D., \& Tinney, C. G. 2005, AJ, 130, 95

Piatek, S., Pryor, C., Bristow, P., Olszewski, E. W., Harris, H. C., Mateo, M., Minniti, D., \& Tinney, C. G. 2006, AJ, 131, 1445

Piatek, S., Pryor, C., Bristow, P., Olszewski, E. W., Harris, H. C., Mateo, M., Minniti, D., \& Tinney, C. G. 2007, AJ, 133, 818

Pompéia, L., et al. 2008, A\&A, 480, 379

Pont, F., Zinn, R., Gallart, C., Hardy, E., \& Winnick, R. 2004, AJ, 127, 840

Prochaska, J. X., Naumov, S. O., Carney, B. W., McWilliam, A., \& Wolfe, A. M. 2000, AJ, 120, 2513

Recchi, S., Matteucci, F., \& D’Ercole, A. 2001, MNRAS, 322, 800

Reddy, B. E., Tomkin, J., Lambert, D. L., \& Allende Prieto, C. 2003, MNRAS, 340,304

Revaz, Y., et al. 2009, A\&A, 501, 189

Ricotti, M., \& Gnedin, N. Y. 2005, ApJ, 629, 259

Robertson, B., Bullock, J. S., Font, A. S., Johnston, K. V., \& Hernquist, L. 2005, ApJ, 632, 872

Romano, D., Chiappini, C., Matteucci, F., \& Tosi, M. 2005, A\&A, 430, 491

Romano, D., Karakas, A. I., Tosi, M., \& Matteucci, F. 2010, A\&A, 522, A32

Romano, D., Tosi, M., \& Matteucci, F. 2006, MNRAS, 365, 75

Ryan, S. G., Norris, J. E., \& Beers, T. C. 1996, ApJ, 471, 254

Sadakane, K., Arimoto, N., Ikuta, C., Aoki, W., Jablonka, P., \& Tajitsu, A. 2004, PASJ, 56, 1041

Saviane, I., Held, E. V., \& Bertelli, G. 2000, A\&A, 355, 56

Sawala, T., Scannapieco, C., Maio, U., \& White, S. 2010, MNRAS, 402, 1599

Sbordone, L., Bonifacio, P., Buonanno, R., Marconi, G., Monaco, L., \& Zaggia, S. 2007, A\&A, 465, 815

Schmidt, M. 1959, ApJ, 129, 243

Schmidt, M. 1963, ApJ, 137, 758

Searle, L., \& Zinn, R. 1978, ApJ, 225, 357

Shetrone, M. D., Bolte, M., \& Stetson, P. B. 1998, AJ, 115, 1888

Shetrone, M. D., Côté, P., \& Sargent, W. L. W. 2001a, ApJ, 548, 592

Shetrone, M. D., Côté, P., \& Stetson, P. B. 2001b, PASP, 113, 1122

Shetrone, M. D., Siegel, M. H., Cook, D. O., \& Bosler, T. 2009, AJ, 137, 62
Shetrone, M. D., Venn, K. A., Tolstoy, E., Primas, F., Hill, V., \& Kaufer, A 2003, AJ, 125, 684

Silk, J., Wyse, R. F. G., \& Shields, G. A. 1987, ApJ, 322, L59

Simon, J. D., Frebel, A., McWilliam, A., Kirby, E. N., \& Thompson, I. B. 2010, ApJ, 716, 446

Smecker-Hane, T. A., Mandushev, G. I., Hesser, J. E., Stetson, P. B., Da Costa, G. S., \& Hatzidimitriou, D. 1999, in ASP Conf. Ser. 192, Spectroscopic Dating of Stars and Galaxies, ed. I. Hubeny, S. R. Heap, \& R. H. Cornett (San Francisco, CA: ASP), 159

Smecker-Hane, T. A., Marsteller, B., Cole, A., Bullock, J., \& Gallagher, J. S. 2009, BAAS, 41, 235

Smecker-Hane, T. A., Stetson, P. B., Hesser, J. E., \& VandenBerg, D. A. 1996, in ASP Conf. Ser. 98, From Stars to Galaxies, ed. C. Leitherer, U. F. Alvensleben, \& J. Huchra (San Francisco, CA: ASP), 328

Smith, G. H. 1984, AJ, 89, 801

Smith, G. H., \& Dopita, M. A. 1983, ApJ, 271, 113

Smith, G. H., Siegel, M. H., Shetrone, M. D., \& Winnick, R. 2006, PASP, 118, 1361

Smith, H. A., \& Stryker, L. L. 1986, AJ, 92, 328

Sohn, S. T., et al. 2007, ApJ, 663, 960

Springel, V., et al. 2008, MNRAS, 391, 1685

Starkenburg, E., et al. 2010, A\&A, 513, A34

Steigman, G. 2007, Annu. Rev. Nucl. Part. Sci., 57, 463

Stephens, A., \& Boesgaard, A. M. 2002, AJ, 123, 1647

Stetson, P. B. 1984, PASP, 96, 128

Strigari, L. E., Bullock, J. S., Kaplinghat, M., Simon, J. D., Geha, M., Willman, B., \& Walker, M. G. 2008, Nature, 454, 1096

Suntzeff, N. B., Mateo, M., Terndrup, D. M., Olszewski, E. W., Geisler, D., \& Weller, W. 1993, ApJ, 418, 208

Tafelmeyer, M., et al. 2010, A\&A, 524, 58

Thornton, K., Gaudlitz, M., Janka, H.-T., \& Steinmetz, M. 1998, ApJ, 500, 95

Timmes, F. X., Brown, E. F., \& Truran, J. W. 2003, ApJ, 590, L83

Tinsley, B. M., \& Larson, R. B. 1979, MNRAS, 186, 503

Tolstoy, E., Hill, V., \& Tosi, M. 2009, ARA\&A, 47, 371

Tolstoy, E., Irwin, M. J., Cole, A. A., Pasquini, L., Gilmozzi, R., \& Gallagher, J. S. 2001, MNRAS, 327, 918

Tolstoy, E., et al. 2003, AJ, 125, 707

Tolstoy, E., et al. 2004, ApJ, 617, L119

Vader, J. P. 1986, ApJ, 305, 669

van den Bergh, S. 1962, AJ, 67, 486

van den Hoek, L. B., \& Groenewegen, M. A. T. 1997, A\&AS, 123, 305

Venn, K. A., Irwin, M., Shetrone, M. D., Tout, C. A., Hill, V., \& Tolstoy, E. 2004, AJ, 128, 1177

Wechsler, R. H., Bullock, J. S., Primack, J. R., Kravtsov, A. V., \& Dekel, A. 2002, ApJ, 568, 52

Weisz, D. R., Skillman, E. D., Cannon, J. M., Dolphin, A. E., Kennicutt, R. C., Jr., Lee, J., \& Walter, F. 2008, ApJ, 689, 160

White, S. D. M., \& Rees, M. J. 1978, MNRAS, 183, 341

Winnick, R. A. 2003, PhD thesis, Yale Univ.

Wolf, J., Martinez, G. D., Bullock, J. S., Kaplinghat, M., Geha, M., Muñoz, R. R., Simon, J. D., \& Avedo, F. F. 2010, MNRAS, 406, 1220

Woo, J., Courteau, S., \& Dekel, A. 2008, MNRAS, 390, 1453

Woosley, S. E., Langer, N., \& Weaver, T. A. 1993, ApJ, 411, 823

Woosley, S. E., \& Weaver, T. A. 1995, ApJS, 101, 181

Zinn, R. 1978, ApJ, 225, 790

Zinn, R. 1981, ApJ, 251, 52

Zinn, R., \& Persson, S. E. 1981, ApJ, 247, 849

Zinn, R., \& Searle, L. 1976, ApJ, 209, 734

Zucker, D. B., et al. 2006, ApJ, 643, L103 